\title{
MODELOS VAR-GARCH Y PORTAFOLIOS DE INVERSIÓN TRINACIONALES EN LOS MERCADOS ACCIONARIOS DEL TLCAN
}

Francisco Javier Reyes Zárate*

Facultad de Economía, Universidad Nacional Autónoma de México

\section{Edgar Ortiz ${ }^{* *}$}

Facultad de Ciencias Políticas y Sociales, Universidad Nacional Autónoma de México

(Recibido 11 de abril 2013, aceptado 27 de junio 2013)

\begin{abstract}
Resumen
Este trabajo emplea la metodología M-VARCH (modelos Value at Risk y modelos GARCH multivariados), la cual presupone un mayor conservadurismo y precisión en la estimación de pérdidas potenciales de portafolios de inversión. La diversificación regional en mercados accionarios, bajo el contexto de la globalización, es trascendental porque presenta oportunidades importantes de altos rendimientos minimizando riesgos, dado el diferente grado de desarrollo y estabilidad de sus mercados. Esto resalta la necesidad de una eficiente administración de riesgos. El estudio es aplicado a los tres principales índices accionarios de los países miembros del Tratado de Libre Comercio de América del Norte (TLCAN): Dow Jones Industrial Average, de Estados Unidos; Toronto Stock Exchange, de Canadá; y el Índice de Precios y Cotizaciones, de México, con 2,086 datos diarios para el periodo del 2000-03-01 al 2007-12-31.
\end{abstract}

Abstract

This document employs the M-VARCH Methodology (Value-at-Risk Model and Multivariate GARCH models), which presuppose greater conservatism and precision on estimating potential losses of investment portfolios. Regional diversification in stock markets is transcendental, in a global context, because it opens up important opportunities of high returns minimizing risks, because the different degree of development and stability from its markets. The study is applied to the North America Trade Free Agreement (NAFTA) member's countries employing their principal stock market indexes: Dow Jones Industrial Average (USA), Toronto Stock Exchange (Canada), and Price and Quotation Index (Mexico), with 2,086 daily dates from 2000-03-01 to 2007-12-31.

Clasificación JEL: C16, C32, G15

Palabras clave: Valor en Riesgo, GARCH Multivariado, Administración de riesgos, TLCAN, Back testing.

* División de Estudios de Posgrado, Facultad de Economía, Universidad Nacional Autónoma de México. 04510, México, D.F. Celular: 04455 32287179. Correo electrónico: pacoeko@gmail.com.

** División de Estudios de Posgrado, Facultad de Economía, Universidad Nacional Autónoma de México. 04510, México, D.F. Celular: 525565829 49. Correo electrónico: edgarfo@unam.mx.

Los autores agradecen las observaciones y sugerencias de Alejandra Cabello, Universidad Nacional Autónoma de México, Christian Bucio, Universidad Autónoma del Estado de México, Unidad Profesional de Huehuetoca, Manuel da Rocha Armada, Universidad de Minho, Portugal y de dos árbitros anónimos. 


\section{Introducción}

La liberalización y desregulación financieras, inducidas por los procesos de globalización económica de las últimas décadas, han favorecido el crecimiento de las inversiones internacionales de cartera. Un rasgo importante de estas inversiones ha sido la combinación de activos tanto de mercados maduros como de mercados emergentes. No obstante, al mismo tiempo la correlación positiva entre los mercados accionarios internacionales, incluyendo los mercados emergentes, se ha incrementado significativamente en las últimas décadas, limitando la viabilidad de construir portafolios óptimos con tenencias de activos internacionales. Aún más, el alto rendimiento que ofrecen los mercados emergentes viene acompañado de mayores niveles de riesgo debido a su sensibilidad a los cambios e incertidumbre recurrentes en los fundamentos de sus economías (González y Mascareñas, 1999, López Herrera, 2006, Ortiz et al., 2007a).

López Herrera et al. (2009) apuntan que las oportunidades de inversión y los beneficios que se obtienen a través de la diversificación son determinados principalmente por tres factores: 1) la dinámica de los rendimientos, que representa oportunidades para obtener mayores beneficios, pero también asumir mayores riesgos; 2) la volatilidad, que implica la presencia de eventos no esperados a corto y largo plazo; y 3 ) la correlación y transmisión de volatilidad entre los mercados accionarios internacionales.

Un patrón de diversificación internacional que ha merecido especial atención son las inversiones regionales; su cercanía geográfica y pautas generalmente afines de intercambio económico facilitan el análisis bursátil y toma de decisiones para la composición de portafolios. En estas circunstancias los beneficios dependen de la correlación esperada entre los rendimientos de los mercados incluidos en el portafolio. Un caso especial de este tipo de inversiones constituyen las inversiones realizadas en los mercados de capital de los países integrantes del bloque del TLCAN.

Evidencia empírica reciente señala que estos mercados aún se caracterizan por una segmentación moderada, aunque con cierta tendencia integrativa pero cambiante, aunado al hecho que la integración en este bloque comprende dos países desarrollados como lo son Estados Unidos y Canadá, y un país emergente como México, lo que también se refleja en el tamaño y asimetrías de sus mercados de valores. Considerando la importancia que estos mercados tienen para el fortalecimiento de sus economías e implicaciones que las inversiones de cartera tienen en la integración del bloque, el presente estudio contempla dos objetivos: (1) construir, para el caso de los mercados de capitales del bloque del TLCAN, portafolios de inversión regionales óptimos aplicando extensiones del modelo de Markowitz $(1952,1959)$ integrando la distribución logística y modelos tipo GARCH; y (2) identificar las pérdidas potenciales a las que están expuestos los inversionistas del bloque del TLCAN, aplicando la metodología de Valor en Riesgo Multivariado (M-VARCH) incorporando modelos GARCH alternativos para estimar las pérdidas potenciales de portafolios regionales. Los resultados obtenidos son validados con la prueba de backtesting de Kupiec (1995).

Las hipótesis subyacentes en los objetivos antes señalados tratan de comprobar que dado el grado de segmentación de los mercados de capital 
de los países miembros del TLCAN es posible construir portafolios óptimos aplicando extensiones del modelo de Markowitz y que la exposición al riesgo de dichos portafolios puede ser mejor medida (y por ende administrada) con una aplicación no tradicional del Valor en Riesgo que contemple el empleo del modelos GARCH multivariados y el valor en riesgo condicional.

El trabajo está organizado en seis secciones. A la presente introducción la segunda sección examina indicadores clave de cambio de los mercados accionarios del TLCAN para el período 2000-2007. La tercera sección detalla la evidencia empírica de investigaciones previas. La cuarta sección presenta la metodología econométrica. Incluye; el modelo de portafolios de Markowitz con su extensión a la distribución logística; los modelos GARCH univariados y multivariados; los modelos de valor en riesgo (VaR), resaltando el valor en riesgo condicional (CVaR). De la combinación de los modelos GARCH y valor en riesgo condicional surge precisamente la novel aplicación GARCH-CVaR, una importante primicia para la investigación y administración del riesgo en México; finalmente el modelo de backtesting de Kupiec con el cual se da cuenta de la solidez de los resultados obtenidos.

La quinta sección presenta y analiza los resultados obtenidos de las aplicaciones econométricas. Finalmente, la sexta y última sección presenta las conclusiones.

\section{Patrones de cambio de los mercados accionarios del TLCAN}

Aunque el mercado de capitales mexicano ha mantenido un crecimiento vigoroso en las últimas décadas, una gran distancia aún separa a este mercado de los correspondientes a sus socios del TLCAN. La gran desigualdad entre estos socios comerciales está enraizada en su asimétrico desarrollo económico. Como se resume en el cuadro 1 el Producto Interno Bruto de México es el menor de la región, registrando en 2007 un monto de $\$ 1,025.58$ billones de dólares, en tanto que el nivel de PIB de Estados Unidos y Canadá fue de US\$14,061.80 y US $\$ 1,424.05$ billones de dólares, respectivamente. Cabe resaltar que la economía con mayor dinamismo fue la canadiense, registrando un crecimiento de 2.38 veces de su nivel original de $\$ 724.21$ billones de dólares; la economía con menor crecimiento fue la de Estados Unidos cuyo cambio fue de 1.413 veces, mientras que la economía mexicana tuvo un cambio de $\$ 628,85$ a $\$ 1,025.58$ billones de dólares, es decir, un crecimiento de 1.63 veces mayor al de Estados Unidos, pero menor que el de Canadá; de todas maneras, la economía de Estados Unidos es por mucho la mayor economía de la región. En 2007, la economía de Canadá representó solo el $10.12 \%$ de la economía de Estados Unidos, y la economía mexicana solo representó el 7.29\% del tamaño de la economía de Estados Unidos. 
Cuadro 1. Características de los mercados de valores del TLCAN (2000-2007)

\begin{tabular}{|c|c|c|c|c|c|c|c|c|}
\hline PIB $/ 1$ & 2000 & 2001 & 2002 & 2003 & 2004 & 2005 & 2006 & 2007 \\
\hline $\begin{array}{l}\text { Rstados } \\
\text { Tinidos }\end{array}$ & $9,951.48$ & $10,256.18$ & $10,642.30$ & $11,142.18$ & $11,867.75$ & $12,638.38$ & $13,398.93$ & $14,051.80$ \\
\hline Canada & 724.91 & 715.44 & 734.65 & 865.9 & 992.23 & $1,133.76$ & $1,278.61$ & $1,424.07$ \\
\hline Mrico & 628.35 & 672.82 & 702.02 & 700.32 & 759.78 & 348.95 & 952.54 & $1,025.58$ \\
\hline \multicolumn{9}{|l|}{ Capitalinadiou } \\
\hline $\begin{array}{l}\text { Pstados } \\
\text { Unidos NYSE }\end{array}$ & $11,534.61$ & $11,006.59$ & $9,015.27$ & $11,328.95$ & $12,707.58$ & $13,310.59$ & $15,421.17$ & $15,650.83$ \\
\hline Can ada TSX & 766.2 & 611.49 & 570.22 & 888.68 & $1,177.52$ & $3,603.98$ & $3,865.00$ & $4,013.65$ \\
\hline Menio BMV & 125.2 & 12526 & 103.94 & 122.53 & 171.4 & 239.13 & 348.35 & 397.72 \\
\hline \multicolumn{9}{|l|}{ Valor a egociad o } \\
\hline $\begin{array}{l}\text { Estados } \\
\text { Unidos NYSF }\end{array}$ & $11,050.05$ & 10.439 .32 & $10,311.16$ & $9,691.34$ & $11,618.15$ & $14,125.29$ & $21,790.60$ & $29,909.99$ \\
\hline Canade TSX & 636.54 & 49.53 & 408.16 & 471.54 & $8,767.12$ & $10,086.74$ & $11,807.49$ & $15,320.13$ \\
\hline Menic BMV & 45.77 & 69.66 & 32.29 & 25.87 & 45.39 & 56.68 & 96.44 & 144.75 \\
\hline \multicolumn{9}{|l|}{$\begin{array}{l}\text { CapitalinadiouPIB } \\
(9 \%)\end{array}$} \\
\hline $\begin{array}{l}\text { Pstados } \\
\text { Unidos NYSE }\end{array}$ & 115.91 & 107.20 & 84.71 & 101.68 & 107.08 & 10632 & 115.09 & 111.30 \\
\hline Canada TSX & 105.70 & 85.47 & 77.62 & 102.63 & 118.67 & 31038 & 302.28 & 281.34 \\
\hline Metio BMV & 19.91 & 18.77 & 14.81 & 17.50 & 22.56 & 28.17 & 36.57 & 38.78 \\
\hline \multicolumn{9}{|l|}{ Nitue ero do canpress } \\
\hline $\begin{array}{l}\text { Pstados } \\
\text { Eanidos NYSF }\end{array}$ & 2,429 & 1999 & 1,394 & 1,342 & 1,834 & 1,818 & 1,829 & 1,852 \\
\hline Canada & 1,379 & 1261 & 1,252 & 3,561 & 2,839 & 2,832 & 2,312 & 2,762 \\
\hline Merico BMV & 173 & 167 & 163 & 158 & 151 & 150 & 132 & 125 \\
\hline
\end{tabular}

Nota: 1) Miles de millones de dólares de Estados Unidos.

Fuente: World Federation Exchange, Annual Report; 2) FMI. Años 2002 a 2007.

La asimetría en el tamaño de las economías se refleja en el tamaño relativo de los mercados bursátiles del bloque norteamericano. El mercado de Estados Unidos es el mayor de los tres. La capitalización de este mercado en 2007 fue de $\$ 15,650.83$ billones de dólares, revelando un crecimiento de 1.335 veces respecto a su nivel original de $\$ 11,534.61$ billones de dólares. Aunque este es el mercado mas grande de la región, resalta el altísimo crecimiento de 5.24 veces del mercado de capitales de Canadá, registrando en 2007 un nivel de capitalización de $\$ 4,013.65$ billones de dólares, esto es, un poco más de la cuarta parte de la capitalización del mercado de Estados Unidos. El mercado de México también tuvo un crecimiento significativo de 3.17 veces; no obstante su capitalización en 2007 correspondió solamente a $2.54 \%$ de la capitalización del mercado de Estados Unidos.

El valor negociado en estos mercados también revela grandes diferencias. En niveles el valor negociado en el mercado de capitales de Estados Unidos es mucho mayor que el de sus socios comerciales. Sin embargo, destaca el espectacular crecimiento del valor negociado en el mercado de capitales de Canadá: 25.65 veces, de 636.54 billones de dólares a 15,320.13 billones de 
dólares, en tanto que el crecimiento del valor negociado en los mercados de Estados Unidos y México fue de 2.7 veces y 3.1 veces, respectivamente.

Finalmente, cabe destacar el reducido y preocupante tamaño de la Bolsa Mexicana de Valores (BMV) en lo que se refiere a su capitalización respecto al PIB y respecto al número de empresas que operan en el mercado. En 2007, la capitalización de la BMV correspondió a solo $38.78 \%$ respecto al PIB, en tanto que en las economías de Estados Unidos y Canadá este coeficiente fue de $111.30 \%$ y $281.84 \%$, revelando nuevamente el dinamismo del mercado accionario de Canadá.

Igualmente preocupante es el reducido tamaño del número de empresas que opera en el mercado accionario mexicano; aún más el número de empresas ha decrecido significativamente de 173 empresas a solamente 125, durante el período analizado. En Estados Unidos también se ha registrado una tendencia decreciente, de 2,479 empresas a 1,852; en Canadá la tendencia ha sido opuesta, registrándose un crecimiento en el número de empresas de 1,379 a 2,762 de 2000 a 2007.

El desigual tamaño y desarrollo de los mercados de capitales del bloque del TLCAN no se refleja en las tendencias de los precios de sus mercados. Como se aprecia en la gráfica 1, y partiendo de una base común (enero 3, 2000=100), resalta el notable crecimiento del índice de precios y cotizaciones (IPC) de la Bolsa Mexicana de valores, particularmente desde 2003, alcanzando en diciembre de 2007 niveles de 417.32 por ciento, esto es, un rendimiento acumulado de 317.32 por ciento. Los mercados accionarios de Canadá y Estados Unidos muestran tendencias menos crecientes. El nivel de precios del mercado accionario de Toronto se incrementó a 157.65 puntos, en tanto que el mercado de Estados Unidos se registró el crecimiento menor a 121.80 puntos. $\mathrm{El}$ alto rendimiento del mercado de valores mexicano resalta lo atractivo de la diversificación internacional. No obstante, esta puede estar acotada por dos importantes hechos: (a) la volatilidad de cada uno de estos mercados y (b) la correlación entre los mismos, problemas que se han exacerbado en las últimas décadas a raíz de la liberalización de los mercados financieros y su creciente integración resultante de la globalización financiera. 
Gráfico 1. Niveles de precios de los principales índices financieros de Canadá, Estados Unidos y México.

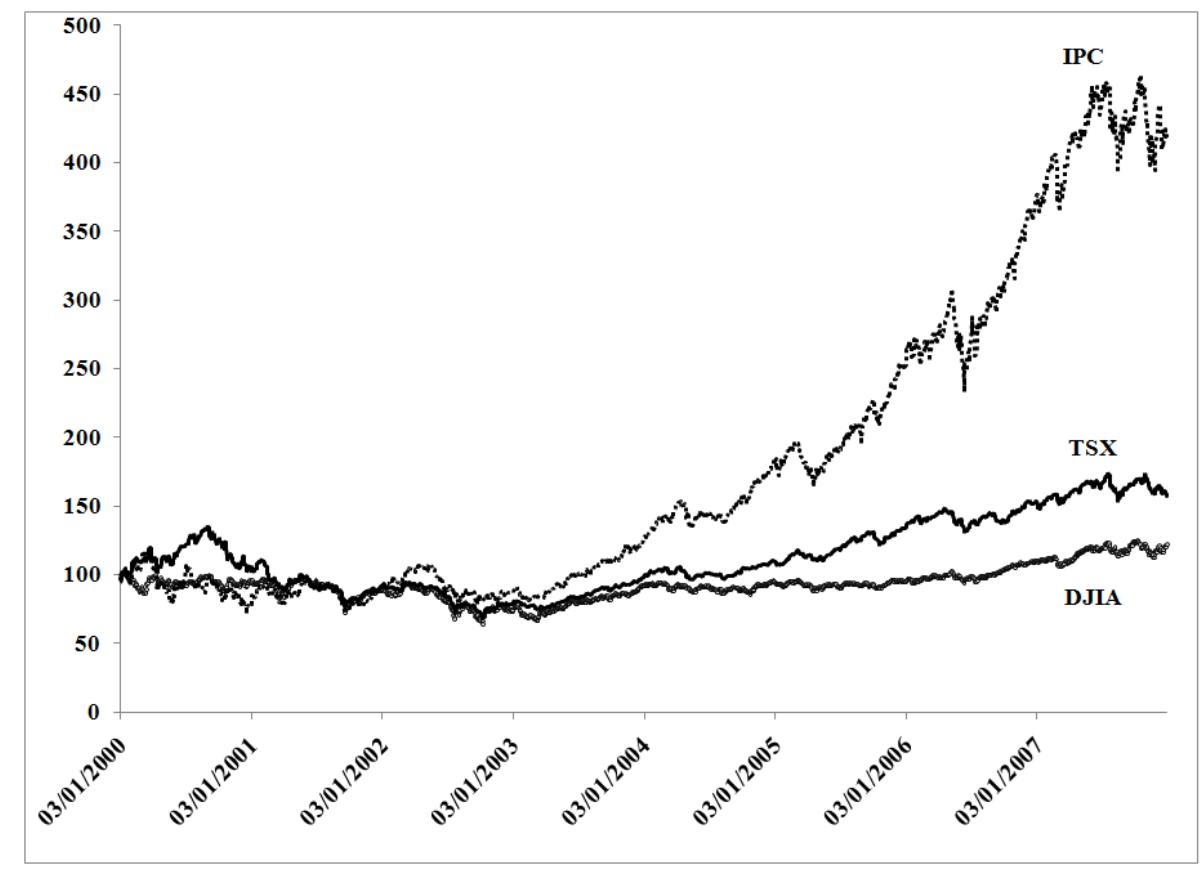

Fuente: Elaboración propia.

México no está alejado de estas tendencias. La gráfica 2 resume los patrones de cambio de los rendimientos de los tres mercados del bloque del TLCAN. En los tres mercados se experimentó una alta volatilidad, la más alta en la Bolsa Mexicana de Valores (BMV) y la más baja en el mercado de capitales de Canadá. En términos anuales, la desviación estándar de estos mercados fue de 0.164 por ciento, 0.152 por ciento y 0.214 por ciento para los casos de Estados Unidos, Canadá y México, respectivamente. Sin embargo puede apreciarse igualmente que la volatilidad de los rendimientos en los tres mercados es dependiente en el tiempo, exhibiendo clusters de volatilidad alta y clusters de volatilidad relativamente estable. Así, la administración del riesgo de portafolios que incluya los índices bursátiles de estos mercados debe tomar en cuenta la heterocedasticidad de sus rendimientos, precisamente una de las preocupaciones principales del presente estudio al estimar las pérdidas potenciales de estos portafolios aplicando la metodología de Valor en Riesgo Multivariado (M-VARCH) incorporando modelos GARCH alternativos. 
Gráfico 2. Evolución de los rendimientos sobre índices accionarios referentes a los mercados financieros en las economías del TLCAN (moneda local)
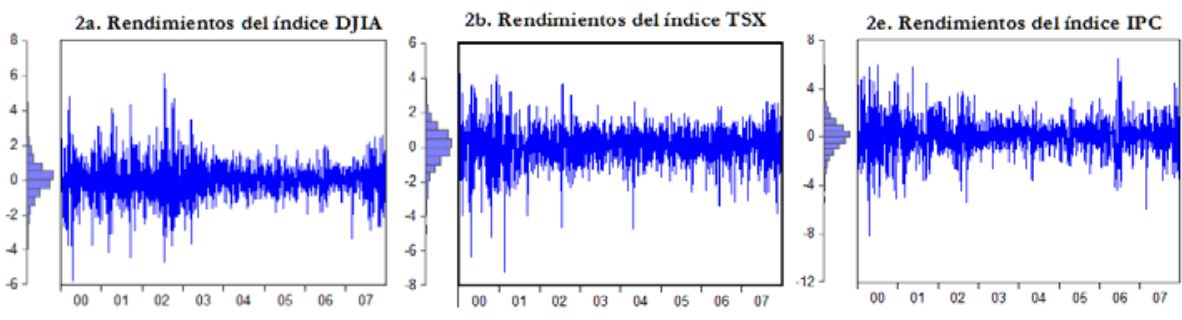

Fuente: Elaboración propia.

En lo que se refiere a la correlación entre los mercados, el cuadro 2 ratifica un hecho mundial: la presencia de correlaciones positivas. Durante el período 2000 a 2007 los mercados accionarios del bloque del TLCAN mostraron una correlación positiva, en todos los casos mayor a 0.50 con un nivel de significancia de 5 por ciento. La correlación mas alta corresponde al caso bilateral de los índices de Estados Unidos y Canadá (0.60); la correlación más baja corresponde a los mercados de Canadá y México (0.51); con el mercado de Estados Unidos, la correlación de la BMV es significativamente alta: 0.56. Estas altas y positivas correlaciones claramente implican la imposibilidad de desarrollar portafolios óptimos conforme al tradicional modelo de Markowitz cuya optimización depende de la presencia de correlaciones positivas y negativas. Esto es, los portafolios regionales del TLCAN además del problema de heterocedasticidad de los rendimientos, sus mercados se caracterizan por una alta correlación positiva entre sí, lo que acota la optimización de la relación riesgo-rendimiento.

Cuadro 2. Correlación entre los rendimientos de índices bursátiles del TLCAN (2000-2007, datos diarios)

\begin{tabular}{|c|c|c|c|}
\hline ÍNDICE & DJIA & TSX & IPC \\
\hline DJIA & 1.00000 & $0.60008^{*}$ & $0.56037^{*}$ \\
\hline TSX & $0.60008^{*}$ & $1.00000^{*}$ & $0.51625^{*}$ \\
\hline IPC & $0.56037^{*}$ & $0.51625^{*}$ & $1.00000^{*}$ \\
\hline
\end{tabular}

*Correlación significativa al nivel de 0.01 por ciento.

\section{Evidencia empírica previa}

Aunque la literatura sobre el desarrollo y aplicación de los modelos VaR y GARCH multivariados se ha enfocado a mercados financieros desarrollados, un creciente e importante número de estudios han abordado el caso de mercados emergentes. Resaltan algunos casos de análisis y aplicación de diferentes metodologías VaR con diferentes criterios de comparación. Tagliafichi (2003) estima el VaR aplicando modelos GARCH $(1,1)$, TARCH $(1,1)$ y EGARCH $(1,1)$ para los índices accionarios BOVESPA de Brasil, MERVAL de Argentina, DJIA de Estados Unidos. Su muestra comprende 735 observaciones, finalizando enero 31 de 2003. El análisis de backtesting comprueba que los resultados son robustos y que cumplen con los ordenamientos 
del acuerdo de Basilea II. Además, resalta el hecho de que aplicando los modelos anteriormente indicados no es necesario utilizar distribuciones con colas gruesas.

Por su parte, Burns (2002) compara estimaciones de VaR empleando modelos GARCH univariados. Su estudio realizado para una muestra del índice S\&P que comprende el período 17 de abril de 1931 a 29 de diciembre de 2000, casi 70 años de rendimientos diarios. Su evidencia revela que con la metodología GARCH se obtienen estimados más precisos que con metodologías tradicionales. Angelidis y Benos (2003) estiman el VaR para portafolios compuestos por los índices S\&P500, Nikkei 225, FTSE 100, CAC 40 y DAX 40 para muestras de $500,100,1,500$ y 2,000 observaciones. Aplican tres interpretaciones diferentes de la familia GARCH: GARCH, TARCH y EGARCH. Su evidencia confirma que estos modelos dan mejores resultados que con modelos que asumen simple normalidad. A los estudios anteriores debe añadirse a Korkmaz y Aydin (2002) quienes aplican modelos EWMA and GARCH para estimaciones diarias de VaR para el índice ISI 30 de la bolsa de valores de Estambul. Su muestra comprende de enero 5 de 1998 a enero 29 de 2000. Sus resultados señalan que estas metodologías mejoran los resultados obtenidos para enero 5, 1998 a enero $29,2002$.

En aplicaciones para el caso de América Latina, Fernández et al. (2002) y Vergara y Maya (2007) presentan evidencia empírica y aplicación de metodologías GARCH paramétricas y no paramétricas para los rendimientos accionarios del mercado colombiano. Alonso y Arcos (2006) emplean varios métodos paramétricos y no paramétricos de VaR para un portafolio representativo de 7 mercados latinoamericanos; EWMA y TGARCH son las metodologías más adecuadas para niveles de confianza de 95\%, pero su desempeño es pobre para niveles de confianza de $99 \%$. Por su parte, aunque no en relación a valores accionarios cabe mencionar el estudio de Gallón y Gómez (2007) por referirse también a América Latina: los autores presentan aplicaciones de modelos VaR y GARCH multivariados aplicados al tipo de cambio del peso colombiano con respecto a monedas duras tales como el dólar americano, el euro, la libra esterlina y el yen japonés; los autores concluyen que la modelación de la distribución condicional para los rendimientos de la tasa de cambio validan la estimación de estos modelos sobre la matriz de covarianza condicional en la determinación de las ganancias obtenidas.

Para el caso de México y su relación con otras economías, de Jesús y Ortiz (2012) utilizan CVARCH y la teoría de valores extremos aplicados a los índices accionarios de los mercados de Brasil y México. López Herrera (2006) presenta un análisis econométrico para el estudio de la prima de riesgo para el mercado mexicano y su relación de segmentación parcial con respecto al Tratado de Libre Comercio de América del Norte (TLCAN); por su parte, Ludlow y Mota (2006) presentan una comparación de volatilidades con los índices Nasdaq y Standard and Poor's 500 (S\&P 500, Estados Unidos), y el índice de Precios y Cotizaciones (IPC, México) mediante una estimación simultánea utilizando modelos GARCH multivariados; por último, Ortiz, et al. (2007b) analizan tanto el proceso de integración de los mercados de capitales de las economías que conforman el bloque regional del TLCAN mediante un modelo econométrico de cointegración, así como también las interrelaciones de volatilidad y rendimientos entre los mercados bursátiles mencionados mediante 
modelos GARCH multivariados. Por último, Ramírez y Ramírez (2007) enfocan su estudio al análisis de metodologías VaR aplicado a acciones mexicanas.

\section{Econometría de la metodología M-VARCH}

\subsection{El modelo de portafolios de Markowitz extendido}

Markovitz (1952, 1959) demostró que la estructura de un portafolio de inversión requiere de conocer el valor esperado y la desviación estándar de los rendimientos del portafolio, cuyos parámetros muestran que el rendimiento del portafolio es igual al rendimiento promedio ponderado de cada uno de los activos que integran un portafolio de inversión; sin embargo, el riesgo (desviación estándar) del portafolio no es equivalente a la desviación estándar ponderada; la desviación estándar de dos variables aleatorias es definida como:

$$
\sigma_{p}=\left[y \sigma^{2}(r)+z \sigma^{2}(s)+2 y z \cdot \operatorname{cov}(a, b)\right]^{\frac{1}{2}}
$$

donde $\sigma_{p}$, es el riesgo (desviación estándar) del portafolio de inversión; $\sigma(r, s)$, es la covarianza de los rendimientos; e $y, z$ son la participación de los activos a y b en el portafolio. Markowitz encuentra un hecho importante. Cuando el portafolio de inversión tiene $N$ activos equiponderados, cada uno con riesgo $\sigma$ y rendimientos no correlacionados, el riesgo del portafolio se define por $\sigma_{p}=$ $\sigma / \sqrt{N}$, es decir, a medida que aumenta $N$, el riesgo del portafolio disminuye. Por otra parte, el rendimiento esperado de un portafolio de inversión está dado por la ecuación (2):

$$
E\left(R_{p}\right)=\sum_{i=1}^{n} W_{i} E\left(R_{1}\right)
$$

En donde $R_{i}$ es el rendimiento de cada activo $p, E_{i}$ es el peso de cada activo en el portafolio, $N$ es el número de activos que participan en el portafolio, y $E\left(R_{p}\right)$ es el rendimiento esperado del portafolio. Así, el riesgo de un Portafolio $P_{f}$ con múltiples alternativas de inversión se logra mediante el cálculo de su desviación estándar:

$$
\sigma_{P_{f}}=\left[\sum_{i=1}^{n} \sum_{j=1}^{n} W_{i} W_{j} C O V_{i j}\right]^{\frac{1}{2}}
$$

Con $\sigma_{p}$ como el riesgo del portafolio. La frontera eficiente del portafolio define el conjunto de portafolios que permiten optimizar el riesgo o la rentabilidad, de tal forma que a cada nivel de rentabilidad se tenga el menor riesgo posible; el agregado de portafolios eficientes puede calcularse resolviendo la ecuación (4):

$$
\operatorname{Min} \sigma_{p}\left(R_{p}\right)=\sum_{i=1}^{n} \sum_{j=1}^{n} X_{i} * X_{j} \sigma_{i j}
$$

s.a

$$
\begin{gathered}
E\left(R_{p}\right) \sum_{i=1}^{n} X_{i} * e\left(R_{i}\right)=V^{*} \\
X_{i} \geq 0(i=1, \cdots, n)
\end{gathered}
$$


Con $X_{i}$ como la proporción del presupuesto del inversionista destinado al activo financiero, $\sigma^{2}\left(R_{p}\right)$ es la varianza del portafolio $p$, y $\sigma_{i j}$ es la covarianza entre los rendimientos de los valores $i$ y $j$.

Así, $E\left(R_{p}\right)$, es la rentabilidad o rendimiento esperado de la cartera $p$, de tal forma que al variar el parámetro $V^{*}$ se obtendrá en cada caso, al resolver (4), el conjunto de proporciones $X_{i}$ que minimizan el riesgo de la cartera, así como su valor correspondiente. La dualidad rentabilidadriesgo de pares $\left[E\left(R_{p}\right), \sigma^{2}\left(R_{p}\right)\right]$ o combinaciones de todas las carteras eficientes se denomina "frontera eficiente". Se deben cumplir simultáneamente dos condiciones: 1) los portafolios o combinaciones de activos deben mostrar mínima varianza dentro de todas las combinaciones que tienen una tasa de rendimiento dada; y 2) deben tener la tasa de rendimientos más alta en todas las posibles combinaciones que tienen una varianza dada.

El modelo de Markowitz tuvo aceptación inmediata pero su aplicación permaneció restringida debido a las limitaciones de los equipos de cómputo de mediados y siguientes tres décadas del siglo pasado. Con el importante desarrollo de la informática así como de la econometría financiera muchos pilares de las finanzas modernas avanzados del siglo pasado han sido sujetos de importantes revisiones y ampliaciones. El modelo de Markowitz ha sido ampliado a fin de sobreponer su supuesto de normalidad de los rendimientos y consiguiente supuesto de volatilidad constante de los rendimientos. Siguiendo recientes avances de la investigación financiera, el presente estudio extiende el Modelo de Markowitz aplicando la distribución logística y modelos de la familia GARCH, para la conformación de portafolios y la medición del valor en riesgo.

\subsection{Ajuste a la distribución logística}

En el caso de los mercados accionarios del bloque del TLCAN, la distribución que mejor se ajusta al comportamiento de sus rendimientos es la distribución logística, lo que permite usar sus parámetros para elaborar modelos y análisis más robustos que aquellos derivados de una distribución normal tradicionalmente empleada en la literatura financiera. Así, los parámetros media-varianza permiten contrastar los resultados obtenidos bajo el supuesto distributivo logístico versus los resultados establecidos en los diferentes modelos GARCH analizados y la posterior cuantificación de los portafolios de inversión mediante el estudio de modelos VaR/CVaR bajo el supuesto distributivo normal.

Como se señaló anteriormente, la distribución logística es semejante a una distribución normal, aunque las colas y el centro están un poco más elevados. Las principales especificaciones de esta distribución son las siguientes: a) parámetros: $(\alpha, \beta)$; dominio en: $x \in(-\infty,+\infty)$; función de densidad (pdf): $\left[\frac{e^{-x-\alpha / \beta}}{\beta\left(1+e^{-x-\alpha / \beta}\right)}\right] ; \mathrm{y}$ función de distribución (cdf): $\left[\frac{1}{\left(1+e^{-x-\alpha / \beta}\right)}\right] ; \mathrm{su}$ media y varianza son, respectivamente: $\mathrm{E}[\mathrm{X}]=\mu \mathrm{y} \operatorname{Var}[\mathrm{X}]=\left[\frac{\pi \beta^{2}}{3}\right]$, este último parámetro, es equivalente a $\sigma^{2}$. La prueba no paramétrica Kolmogorv-Smirnov (K-S) permite comprobar el ajuste de un conjunto de datos sujetos a diferentes distribuciones sobre las cuales se determina la bondad de ajuste de la distribución teórica de probabilidad.

La hipótesis nula consiste en probar si el conjunto de observaciones se ajusta a una distribución $X$. El cuadro 3 detalla el tipo de ajuste de distribución para 
cada una de las series analizadas, en las tres divisas del bloque. A excepción de los indicies TSX e IPC, en dólares de Estados Unidos la distribución ajustada permanece como normal; las demás series se ajustan a una distribución logística. Es posible observar un incremento en el promedio de los rendimientos en comparación con la distribución real (la cual asume normalidad); i.e., el promedio del rendimiento del índice canadiense se incrementa de 0.009 a 0.028 por ciento diario, el índice norteamericano se incrementa de 0.022 a 0.031 por ciento, y el índice mexicano se contrae levemente de 0.069 a 0.061 por ciento. Por otra parte, la volatilidad se ve reducida para los tres índices financieros de tal manera que para el caso canadiense disminuye de 1.03 a 0.92 por ciento, para EE.UU. de 0.95 a 0.84 por ciento y para el caso mexicano se observa una disminución de 1.35 a 1.14 por ciento. En suma, empleando la distribución logística, en el caso del presente estudio se obtiene una mayor precisión en los parámetros estadísticos y estableciendo en el modelo de Markowitz un conjunto más refinado de covarianzas para la optimización de portafolios.

Cuadro 3. Ajuste de distribución de las series de rendimientos de índices financieros TSX, DJIA e IPyC homologados a cada divisa del TLCAN.

\begin{tabular}{|c|c|c|c|c|c|c|c|c|}
\hline \multicolumn{2}{|c|}{$\begin{array}{r}\text { Nombre de la } \\
\text { Variable* }\end{array}$} & TSX_EU & \multicolumn{2}{|c|}{$\begin{array}{l}\text { Nombre de la } \\
\text { Variable* }\end{array}$} & DJIA_EU & \multicolumn{2}{|c|}{$\begin{array}{r}\text { Nombre de la } \\
\text { Variable* }\end{array}$} & IPC_EU \\
\hline \multicolumn{2}{|c|}{ Mejor Supuesto } & 0.00040 & \multicolumn{2}{|c|}{ Mejor Supuesto } & 0.00009 & \multicolumn{2}{|c|}{ Mejor Supuesto } & 0.00061 \\
\hline \multicolumn{2}{|c|}{$\begin{array}{r}\text { Distribución } \\
\text { Ajustada }\end{array}$} & Normal & \multicolumn{2}{|c|}{$\begin{array}{r}\text { Ajustado } \\
\text { Distribución }\end{array}$} & Logística & \multicolumn{2}{|c|}{$\begin{array}{r}\text { Ajustado } \\
\text { Distribución } \\
\text { Ajustada }\end{array}$} & Normal \\
\hline & & 0.00063 & \multirow{3}{*}{\multicolumn{2}{|c|}{$\begin{array}{r}\alpha \\
\beta \\
\text { Estadístico K-S }\end{array}$}} & 0.00028 & \multirow{3}{*}{\multicolumn{2}{|c|}{$\begin{array}{r}\alpha \\
\beta \\
\text { Estadístico K-S }\end{array}$}} & 0.00067 \\
\hline & $\beta$ & 0.00967 & & & 0.00508 & & & 0.01244 \\
\hline \multicolumn{2}{|c|}{ Estadístico K-S } & 0.02436 & & & 0.02084 & & & 0.03237 \\
\hline \multicolumn{2}{|r|}{ P-Value } & 0.16598 & \multicolumn{2}{|r|}{ P-Value } & 0.32262 & \multicolumn{2}{|r|}{$P$-Value } & 0.02471 \\
\hline \multirow{3}{*}{ Media } & Real & Teórica & \multirow[b]{2}{*}{ Media } & Real & Teórica & \multirow[b]{2}{*}{ Media } & Real & Teónica \\
\hline & 0.00040 & 0.00063 & & 0.00009 & 0.00028 & & 0.00061 & 0.00067 \\
\hline & 0.01089 & 0.00967 & \multirow[t]{2}{*}{ Desv. est. } & 0.01038 & 0.00922 & \multirow[t]{2}{*}{ Desv.est. } & 0.01518 & 0.01244 \\
\hline & - & & & & & & - & \\
\hline Asimetr & 0.55366 & 0.00000 & Asimetría & 0.05988 & 0.00000 & Asimetría & 0.10681 & 0.00000 \\
\hline Curtosis & 2.88927 & 0.00000 & Curtosis & 3.18326 & 1.20000 & Curtosis & 3.04164 & 0.00000 \\
\hline
\end{tabular}


140 Nueva Época REMEF (The Mexican Journal of Economics and Finance)

Cuadro 3. Ajuste de distribución de las series de rendimientos de índices financieros TSX, DJIA e IPyC homologados a cada divisa del TLCAN (continuación)

Pane1 B. Ajuste distribucional de rendimientos de las series en dólares (Canadá)

\begin{tabular}{|c|c|c|c|c|c|c|c|c|}
\hline \multicolumn{3}{|c|}{$\begin{array}{l}\text { Nombre de la } \\
\text { Variable* TSX CN }\end{array}$} & \multicolumn{3}{|c|}{ Nombre de la } & & Nombre de la & IPC_CN \\
\hline \multicolumn{2}{|c|}{ Mejor Supuesto } & & \multicolumn{2}{|c|}{ Mejor Supuesto } & & \multicolumn{2}{|c|}{ Mejor Supuesto } & \\
\hline \multicolumn{2}{|c|}{ Ajustado } & 0.00022 & \multicolumn{2}{|c|}{ Ajustado } & -0.00009 & \multicolumn{2}{|c|}{ Ajustado } & 0.00043 \\
\hline \multicolumn{2}{|c|}{ Distribución } & Logística & \multicolumn{2}{|c|}{$\begin{array}{r}\text { Distribución } \\
\text { Ajustada }\end{array}$} & Logística & \multicolumn{2}{|c|}{$\begin{array}{r}\text { Distribución } \\
\text { Ajustada }\end{array}$} & Logística \\
\hline & $\alpha$ & 0.00031 & \multirow{3}{*}{\multicolumn{2}{|c|}{ Estadístico K-S }} & -0.00009 & \multirow{3}{*}{\multicolumn{2}{|c|}{$\begin{array}{r}\alpha \\
\beta \\
\text { Estadístico K-S }\end{array}$}} & 0.00045 \\
\hline & & 0.00463 & & & 0.00595 & & & 0.00749 \\
\hline \multicolumn{2}{|c|}{ Estadístico K-S } & 0.02441 & & & 0.02520 & & & 0.02520 \\
\hline \multicolumn{2}{|r|}{$P$-Value } & 0.16450 & \multicolumn{2}{|r|}{$P$-Value } & 0.13954 & & P-Value & 0.13954 \\
\hline \multirow[b]{2}{*}{ Media } & Real & Teórica & \multirow[b]{2}{*}{ Media } & Real & Teórica & \multirow[b]{2}{*}{ Media } & Real & Teórica \\
\hline & 0.00022 & 0.00031 & & -.00009 & -.00009 & & 0.00043 & 0.00045 \\
\hline Desv.est. & 0.00958 & 0.00840 & Desv. est. & 0.01122 & 0.01079 & Desv.est. & 0.01538 & 0.01358 \\
\hline \multirow{2}{*}{$\begin{array}{r}\text { Asimetria } \\
\text { Curtosis }\end{array}$} & 0.48388 & 0.00000 & \multirow{2}{*}{$\begin{array}{l}\text { Asimetría } \\
\text { Curtosis }\end{array}$} & & 0.00000 & \multirow{2}{*}{ Asimetría } & & 0.00000 \\
\hline & 3.67515 & 1.20000 & & 2.13557 & 1.20000 & & 2.68083 & 1.20000 \\
\hline
\end{tabular}

Panel C. Ajuste distribucional de rendimientos de las series en pesos (México)

\begin{tabular}{|c|c|c|c|c|c|c|c|c|}
\hline \multicolumn{2}{|c|}{$\begin{array}{r}\text { Nombre de la } \\
\text { Variable* }\end{array}$} & TsX_MX & \multicolumn{2}{|c|}{ Nombre de la } & DJIA_MX & $\begin{array}{r}\text { Nomb } \\
\mathrm{V} \\
\end{array}$ & $\begin{array}{l}\text { bre de la } \\
\text { Jariable* }\end{array}$ & IPC_MX \\
\hline \multicolumn{2}{|c|}{ Mejor Supuesto } & 0.00 & \multicolumn{2}{|c|}{ Mejor Supuesto } & 0.00017 & \multicolumn{2}{|c|}{ Mejor Supuesto } & 0.00069 \\
\hline Dist & $\begin{array}{l}\text { ribución } \\
\text { Ajustada }\end{array}$ & \begin{tabular}{|l|} 
Logística \\
\end{tabular} & \multicolumn{2}{|c|}{$\begin{array}{l}\text { Ajustado } \\
\text { Distribución }\end{array}$} & \begin{tabular}{|l|} 
Logística \\
\end{tabular} & \multicolumn{2}{|c|}{$\begin{array}{r}\text { Distribución } \\
\text { Ajustada }\end{array}$} & Logística \\
\hline & $\alpha$ & 0.00071 & \multirow{2}{*}{\multicolumn{2}{|c|}{$\begin{array}{l}\alpha \\
\beta\end{array}$}} & 0.00014 & \multicolumn{2}{|c|}{$\alpha$} & 0.00061 \\
\hline & $\beta$ & 0.00559 & & & 0.00528 & \multirow{2}{*}{\multicolumn{2}{|c|}{$\begin{array}{r}\beta \\
\text { Estadístico K-S }\end{array}$}} & 0.00633 \\
\hline \multicolumn{2}{|c|}{ Estadístico K-S } & 0.01721 & \multicolumn{2}{|c|}{ Estadístico K-S } & 0.01997 & & & 0.02705 \\
\hline \multicolumn{2}{|r|}{$P$-Value } & 0.56378 & & $P$-Value & 0.37305 & \multicolumn{2}{|c|}{ P-Value } & 0.09303 \\
\hline \multirow[b]{2}{*}{ Media } & Real & Teórica & \multirow[b]{2}{*}{ Media } & Real & Teórica & \multirow[b]{2}{*}{ Media } & Real & Teórica \\
\hline & 0.00047 & 0.00071 & & 0.00017 & 0.00014 & & 0.00069 & 0.00061 \\
\hline Desv.est. & 0.01070 & 0.01015 & Desv. est. & 0.01061 & 0.00957 & Desv. est. & 0.01351 & 0.01148 \\
\hline Asir & & 0.00 & & & & & & \\
\hline Curtosis & 2.59315 & 1.20000 & Curtosis & 2.63868 & 1.20000 & Curtosis & 2.81947 & 1.20000 \\
\hline
\end{tabular}

*Notas: Nomenclaturas de los diferentes rendimientos expresados en moneda local: A) TSX_EU (índice canadiense), DJIA_EU (índice americano), y IPC_EU (índice mexicano); B) TSX_CN (índice canadiense), DJIA_CN (índice americano), y IPC_CN (índice mexicano); C) TSX_CN (índice canadiense), DJIA_MX (índice americano), y RIPC_MX (índice mexicano). Los valores del inciso a) se expresan en dólares americanos, los del inciso b) en dólares canadienses y los del inciso c) en pesos mexicanos. Fuente: elaboración propia. Mediante el uso de RiskSimulator. 


\subsection{Modelos univariados GARCH}

El análisis de riesgo y la incertidumbre en los mercados financieros han aumentado las técnicas que permiten modelar las dependencias temporales en la varianza. El modelo GARCH(1,1) de Bollerslev (1986) está basado en el supuesto de que los pronósticos de la varianza cambiante en el tiempo dependen de la varianza rezagada del activo. Un incremento o un decremento inesperado en el rendimiento en el tiempo $t$ generará un incremento en la variabilidad esperada del siguiente periodo.

$$
\sigma_{t}^{2}=\omega+\alpha \varepsilon_{t-1}^{2}+\beta \sigma_{t-1}^{2}
$$

En la ecuación $5, \alpha$ es el promedio, $\varepsilon_{t-1}^{2}$ son las noticias sobre la volatilidad del periodo anterior (el término $\mathrm{ARCH}$ ), $\sigma_{t-1}^{2}$ es la varianza pronosticada del segundo periodo (el término GARCH).

El modelo TARCH (Threshold ARCH) está basado en el supuesto de que los cambios no esperados en el rendimiento de los activos tienen diferentes efectos sobre la varianza condicional. Un incremento inesperado es presentado cuando surgen las buenas noticias y contribuyen a la varianza con el multiplicador $\alpha$. Por el contrario, un decremento inesperado representa malas noticias y contribuye a la varianza con el multiplicador $\alpha+\gamma$. La fórmula matemática del modelo es la siguiente (ecuación 6):

$$
\sigma_{t}^{2}=\omega+\alpha \varepsilon_{t-1}^{2}+\gamma \varepsilon_{t-1}^{2} d_{t-1}+b \sigma_{t-1}^{2}
$$

donde las buenas noticias $\left(\varepsilon_{t}>0\right)$, o las malas noticias $\left(\varepsilon_{t}<0\right)$, tienen efectos diferentes sobre las varianzas condicionales: $d_{t-1}=1 \mathrm{si} \varepsilon_{t-1}<0$ y $d_{t-1}=0 \mathrm{si}$ $\varepsilon_{t-1}>0$.

El modelo de volatilidad dinámica o con suavización exponencial (conocido como EWMA, por sus siglas en inglés, Exponential Weighted Moving Average) asigna mayor peso a las estimaciones históricas más recientes de una serie de tiempo, presentando la ventaja de que la volatilidad dinámica captura más rápido las variaciones fuertes de precios en los mercados con respecto a un promedio simple (o volatilidad histórica) y por ello es posible obtener mejores pronósticos cuando existe volatilidad alta. Su modelo se expresa como:

$$
\sigma_{t}^{2}(1-\lambda) \sum_{i=1}^{T} \lambda^{i-1} r_{t-i}^{2}
$$

El modelo (ecuación 7) depende de un factor de caída (decay factor), con $0<$ $\lambda<1$. Este parámetro asigna los pesos aplicados a las observaciones de tal manera que, mientras más pequeño sea $\lambda$, mayor peso se asignará a los datos recientes.

\subsection{Procesos multivariados GARCH}

La volatilidad de las series univariadas ha sido objeto en las últimas décadas de múltiples investigaciones; avanzando en fronteras de la economía financiera, actualmente ha adquirido gran relevancia la modelación de co-movimientos de varias series, debido a que muestran dependencia temporal o contemporánea, 
observándose como componentes de un vector-valuado (multivariado). De esta manera, es importante reconocer la naturaleza del modelo GARCH multivariado. Por ejemplo, la valuación de activos y la administración de riesgos dependen crucialmente de la estructura de la covarianza condicional de los activos de un portafolio. La especificación de los modelos GARCH no sugiere una extensión natural para el marco multivariado. Además, la expectativa (condicional) de un vector de tamaño $m$ es un vector de tamaño $m$, pero la varianza (condicional) es una matriz $m \times m$ (Bauwens et al., 2006).

En el modelo VECH general, cada elemento de $H_{t}$ es una función lineal de errores cuadrados rezagados y productos cruzados de los valores de los errores y rezagos de los elementos de $H_{t}$. El modelo es definido como

$$
\begin{gathered}
h_{t}=c+A \eta_{t-1}+G h_{t-1} \\
h_{t}=\operatorname{vech}\left(\mathbf{H}_{\mathbf{t}}\right) \\
\eta_{t-1}=\operatorname{vech}\left(\varepsilon_{\mathbf{t}} \varepsilon_{\mathbf{t}}{ }^{\prime}\right)
\end{gathered}
$$

vech(.) es el operador que conjunta la porción triangular más baja de una matriz $N \times N$ como un vector $N(N+1) / 2 \times 1$. $A$ y $G$ representan las matrices de parámetros cuadrados de orden $(N+1) / 2$ y $c$ es un vector de parámetros $(N+1) N / 2 \times 1$.

Debido a la problemática que presenta este modelo, solamente aplicado en la práctica para el caso bivariado, Bollerslev et al. (1988) sugieren el modelo VECH diagonal (DVECH) en el cual las matrices $A$ y $G$ se asumen diagonales, y cada elemento $h_{i j t}$ depende solamente de su rezago propio y del valor previo de $\varepsilon_{i t} \varepsilon_{j t}$.

En particular, se definen las matrices $N \times N$ simétricas, $\mathrm{A}^{\circ}, \mathrm{G}^{\circ} \mathrm{yC}^{\circ}$, como las matrices implícitas por las relaciones $\mathrm{A}=\operatorname{diag}\left[\operatorname{vech}\left(\mathrm{A}^{\mathrm{o}}\right)\right]$, $\mathrm{G}=\operatorname{diag}\left[\operatorname{vech}\left(\mathrm{G}^{\circ}\right)\right]$ y $\mathrm{c}=\operatorname{vech}\left(\mathrm{C}^{\circ}\right)$. El modelo diagonal puede escribirse así:

$$
\mathbf{H}_{\mathbf{t}}=\mathbf{C}^{\mathbf{o}}+\mathbf{A}^{\mathbf{o}} \odot\left(\varepsilon_{\mathbf{t}-\mathbf{1}} \varepsilon_{\mathbf{t}-\mathbf{1}}^{\prime}\right)+\mathbf{G}^{\mathbf{o}} \odot \mathbf{H}_{\mathbf{t}-\mathbf{1}}
$$

Para sobreponer la dificultad para garantizar la positividad de la matriz $H_{t}$ en el modelo VECH sin imponer fuertes restricciones sobre los parámetros, Engle y Kroner (1995) proponen una nueva parametrización para $H_{t}$ que impone fácilmente su positividad, esto es, el modelo D-BEKK $(1,1, \mathrm{~K})$, el cual es definido como

$$
\begin{gathered}
\varepsilon_{t-1} \varepsilon_{t-1}^{\prime} A_{k}^{*}+\sum_{k=1}^{k} G_{k}^{*^{\prime}} H_{t-1} G_{k}^{*} \\
H_{t}=C C^{\prime}+\sum_{k=1}^{k} \sum_{j=1}^{k} A^{\prime} \varepsilon_{t-1} \varepsilon_{t-1} A_{j k} \sum_{k=1}^{k} \sum_{j=1}^{p} G_{j k}^{\prime} H_{t-j} G_{j k}
\end{gathered}
$$

Donde $C, A$ y $G$ son matrices $N \times N$ pero $C$ es triangular superior. El límite de la sumatoria $k$ determina la generalidad del proceso. Los parámetros del modelo $B E K K$ no representan directamente el impacto de los diferentes términos rezagados sobre los elementos de $H_{t}$, como el modelo $V E C H$. 
El modelo $B E K K$ es un caso especial del modelo $V E C H$. Uno de los principales problemas al estimar un modelo $V E C H$ y un modelo $B E K K$ es el alto número de parámetros desconocidos (incluso después de imponer varias restricciones). Por esta razón el uso de estos modelos se limita al análisis de un máximo de tres a cuatro series. Los modelos factoriales y ortogonales pretenden contrarrestar esta dificultad imponiendo una estructura dinámica común en todos los elementos de $H_{g}$, lo cual resulta en modelos con menos parámetros. Bollerslev (1990) propone una clase de modelo $M G A R C H$ en el cual las correlaciones condicionales son constantes y así las covarianzas condicionales son proporcionales al producto de las desviaciones estándar condicionales correspondientes. Esta restricción reduce el número de parámetros desconocidos y simplifica la estimación. El modelo $C C C$ es definido como:

$$
H_{t} D_{t} R D_{t}=\left(\rho_{i j} \sqrt{h_{i i t} h_{j j t}}\right)
$$

donde

$$
D_{t}=\operatorname{diag}\left(h_{11 t}^{\frac{1}{2}} \cdots h_{N N t}^{\frac{1}{2}}\right)
$$

$H$ it puede ser definido como un modelo GARCH invariado, y $R=\left(\rho_{i j}\right)$ es una matriz definida positiva simétrica con $\rho_{i j}=1 \forall i$. $R$ es la matriz que contiene las correlaciones condicionales constantes $\rho_{i j}$.

\subsection{Medidas de valor en riesgo (Var) y Var condicional (Cavar)}

El Var se define como la máxima pérdida esperada para una probabilidad dada sobre un periodo de tiempo establecido, y desde luego puede ser tratado como una medida de riesgo asociada con eventos raros acontecidos en los mercados financieros. La medida no es exacta debido a que está asociada a un nivel de confianza, obligando así a la aplicación de probabilidades de sufrir pérdidas superiores. De esta manera, el número $V a R_{1-q}^{X}$ es una medida estadística que expresa el peor valor de $X$ con un cierto nivel de confianza en un intervalo de tiempo dado con cierto nivel de confianza. La cuantificación del Var puede ser estimada mediante métodos paramétricos y no paramétricos (destacando para el segundo caso el método de simulación histórica). En este trabajo se aplicará la metodología paramétrica que abarca el cálculo de la matriz de varianza-covarianza (delta-normal). De esta manera, el Var de portafolio es simplemente una constante dependiente del nivel de confianza $\alpha$, multiplicada por la raíz del producto de volatilidad y las ponderaciones del portafolio, así como el factor de agregación/desagregación del tiempo $\sqrt{\Delta t}$ :

$$
V a R \equiv \alpha \sqrt{\left[W^{T}\right]\left[\sum\right][W]} \cdot \sqrt{\Delta t}
$$

Donde $\Sigma=[\sigma][\mathrm{C}][\sigma] . \boldsymbol{\Sigma}$ es la matriz de varianza-covarianza, la cual incluye las correlaciones entre los factores que afectan el valor del portafolio $(n \times n) ; \sigma$ es la matriz diagonal de volatilidades de los cambios porcentuales de los factores de riesgo; y $\mathbf{C}$ es la matriz de correlaciones.

Existen serias críticas al modelo $V a R$ en cuanto a que demuestra inestabilidad y dificultad para trabajar de forma numérica si no existe una distribución normal de pérdidas, como lo exhibe la evidencia empírica. De esta manera, la coherencia está únicamente basada en la desviación 
estándar de distribuciones normales sobre los rendimientos de los activos, ya que bajo los supuestos de la distribución normal el $V a R$ es proporcional a la desviación estándar de los rendimientos de los instrumentos. Las contribuciones demuestran que el VaR posee características matemáticas no deseables (subaditividad y convexidad). Uryasev y Rockafellar (2002) dieron una respuesta a la problemática mencionada con el Conditional Value at Risk $(C V a R)$. De forma particular, cuando la distribución de pérdidas y ganancias se asume como una distribución normal, el $C V a R$ se cuantifica de la manera siguiente:

$$
(C \operatorname{VaR})_{\varphi}(X)=E\left[-X \mid-X \leq \operatorname{VaR}_{\varphi}(X)\right]=\frac{e^{\frac{-q^{2} \varphi}{2}}}{\varphi \sqrt{2 \pi}}
$$

\subsection{Test de proporción de fallas de Kupiec}

Esta prueba propuesta por Kupiec (1995) tiene como propósito cuantificar si el nivel significativo propuesto por el modelo $V a R$ es consistente con la proporción de fallas que el modelo presenta, es decir, se trata de verificar si el modelo es adecuado contando las veces que las pérdidas o ganancias exceden al $V a R$ durante un periodo. La hipótesis a verificar trata de validar si la proporción de fallas es similar a la significancia estadística del modelo. De esta manera se verifica la probabilidad de que el número de fracasos sea igual a " $x$ " sobre una muestra " $n$ " se determine a partir de una distribución binomial $P\left(x ; n ; p^{*}\right)=\left(\begin{array}{c}n \\ x\end{array}\right)\left(p^{*}\right)\left(1-p^{*}\right)^{n-x}$. La probabilidad de fracaso $\left(p^{*}\right)$ de los modelos $V a R$ se estima en la práctica a partir de un proceso de máxima verosimilitud (Likelihood Ratio, siglas LR). Finalmente, la estimación consiste en tomar logaritmos de la distribución binomial y maximizar esta función con respecto a la probabilidad estimada $(\tilde{p})$. Al obtenerse el estimador $\mathbf{L R}$ se debe establecer un comparativo estadístico entre las probabilidades teórica y estimada ( $p^{*}$ y $\tilde{p}$, respectivamente). Para la evaluación de la significancia se usa la razón de máxima verosimilitud, obtenido a partir del logaritmo de la distribución de probabilidad aplicada para cada una de estas probabilidades, la razón de verosimilitud se define como:

$$
L R_{U C}=2\left[\frac{(p *)^{x}\left(1-p^{*}\right)^{n-x}}{\tilde{p}^{x}(1-\tilde{p})^{n-x}}\right]
$$

En esta razón no se toma en cuenta la combinatoria ya que se simplifica al considerarse en el numerador y el denominador. La prueba $\mathbf{L R}$ representa una distribución Chi- cuadrada con 1 grado de libertad.

\section{Datos y estimación de los modelos}

\subsection{Datos}

Un atractivo muy importante para un inversionista es la diversificación, en particular si la diversificación está basada en activos financieros de distintos países. En el presente estudio se utilizan índices accionarios, práctica ineludible en la literatura financiera, porque resumen la conducta cotidiana de los principales valores que participan las bolsas nacionales. En el presente 
estudio un inversionista de cada país del bloque del TLCAN invierte en su moneda local o internacionalmente en cualquiera de las divisas de los otros socios del bloque. Así, la inversión inicial así como su ganancia/pérdida se miden en la moneda local del país en donde se realiza la inversión: dólar norteamericano, dólar canadiense y peso mexicano.

La aplicación de la metodología M-VARCH se realiza con los índices: 1) Toronto Stock Exchange (TSX, Canadá); 2) Dow Jones International Average (DJIA, EE.UU.); y 3) Índice de Precios y Cotizaciones (IPC, México). El periodo de análisis de estudio abarca del 3 de enero de 2000 al 31 de diciembre de 2007, contando con un total de 2,086 datos diarios para cada índice propuesto.

\subsection{Análisis econométricos}

Para la realización de los diferentes portafolios de inversión bajo el enfoque de Markowitz, mediante la transformación de la matriz de varianza-covarianza, proporcional al número de activos que componen los portafolios de inversión trinacionales. Con el fin de obtener resultados coherentes en los resultados de los portafolios de inversión y los modelos econométricos aplicados para la metodología propuesta, los índices son homologados entre sí con los respectivos tipos de cambio de las demás economías participantes, a una base común de 100. La tasa diaria de rendimiento corresponde al porcentaje del logaritmo de la razón del rendimiento, esto es, $R_{i}=\ln \left(P_{t}^{*}\left(1 / P_{t-1}\right)\right)$, donde $R_{i}$ es la tasa de rendimiento, $P_{t}$ es el precio de cierre en el día $t$ y el día $t-1$, y $t$ es el intervalo de tiempo.

El cuadro 4 muestra las estadísticas básicas. Se aprecian diferencias en los promedios de los rendimientos y las desviaciones estándar de los tres índices pertenecientes a cada mercado. Además, se puede apreciar que la inversión en el índice DJIA en dólares de Estados Unidos y en pesos presenta el riesgo más bajo (cuantificado por su desviación estándar), pero sus rendimientos (cuantificados por su media simple) también son los más bajos en cualquiera de los tres países en donde se desee realizar la inversión. Incluso, en Canadá este índice llega a ser negativo, lo cual podría explicarse por el bajo atractivo que representó el comportamiento conservador del índice norteamericano en comparación con otros índices de mercados internacionales, por lo cual el inversionista financiero canadiense podría haber optado por invertir en otros activos más atractivos. La apuesta por la inversión en el IPC mexicano en pesos o su transformación en dólares americanos o canadienses, en cambio, presentan la tasa de rendimiento más alta al igual que la volatilidad. Durante esta etapa es posible que el inversionista asuma una posición agresiva y pueda obtener mayores ganancias desde el punto de vista especulativo. 
146 Nueva Época REMEF (The Mexican Journal of Economics and Finance)

Cuadro 4. Estadísticas básicas. Series de rendimientos logarítmicos

\begin{tabular}{|c|c|c|c|c|c|c|c|c|c|}
\hline Mone da & Variable & Media & & Mediana & Máximo & Mínimo & Sesgo & Curtosis & \\
\hline \multirow{3}{*}{$\begin{array}{l}\text { Rdos. } \\
\text { Dólares } \\
\text { EE.UU. }\end{array}$} & $X_{-} E U$ & .00040 & 0.01089 & 00118 & 0.04265 & -0.07223 & & & 826 \\
\hline & $D J I A_{-} E U$ & & .01038 & & & & & & \\
\hline & $I P C_{-} E U$ & & & 0 & & -0.09 & & & \\
\hline \multirow{3}{*}{$\begin{array}{l}\text { Rdos. } \\
\text { Dólares } \\
\text { Can }\end{array}$} & & 000 & 0958 & 1 & & & & & \\
\hline & & & & & & & & & \\
\hline & & & & & & & & & \\
\hline \multirow{3}{*}{$\begin{array}{c}\text { Rdøs. } \\
\text { Pesos } \\
\text { mexicanos }\end{array}$} & $T S X \_M X$ & & & & & & & & \\
\hline & $D J A_{-} M X$ & & & 0.00026 & & & & & 601.85 \\
\hline & IPC_MX & 0.00069 & 0.01351 & 0.00112 & 0.06510 & -0.08267 & -0.13445 & 5.80984 & 692.17 \\
\hline
\end{tabular}

Fuente:Elaboración propia

Como es de esperarse, y corroborando los hechos estilizados, no hay evidencia de la existencia de normalidad de la rentabilidad diaria. De hecho, en los tres mercados se observa el efecto clustering o agrupamiento de datos, como se señaló anteriormente (figura 2). Los cambios en el nivel de volatilidad implican la existencia de varianza condicional no constante (volatility clustering).

En el cuadro 5 se aprecian los resultados de la estimación de la media y la varianza de los portafolios en dólares americanos, en dólares canadienses y en pesos mexicanos, respectivamente. El panel A muestra los modelos univariados; panel B presenta los modelos multivariados. La selección de optimación sobre los parámetros de los modelos GARCH está basada en los criterios de máxima verosimilitud (MV), Akaike (AIC) y Schwarz (SIC). Se observa el número de parámetros resultantes en los modelos M-GARCH sujetos al presente análisis. Es posible apreciar que los portafolios construidos por la desviación estándar y el método EWMA resultan tener mayores desventajas en la optimación de parámetros porque se sitúan como los más altos para el caso del parámetro de riesgo y de los más bajos para el caso del rendimiento. 
CUADRO 5. Optimización de los portafolios de inversión en los países del TLCAN (modelos M-VARCH).(Método de optimización lagrangiano de Markowitz). Porcentaje diario.

\begin{tabular}{|c|c|c|c|c|}
\hline $\begin{array}{c}\text { Portafolio } \\
\text { Optimiz ado } \\
\text { (Markovitz)/ } \\
\text { Mode bs: }\end{array}$ & Parámetro & $\begin{array}{l}\text { Portafolio } 1 \\
\text { US dólares } \\
\text { (por ciento) }\end{array}$ & $\begin{array}{c}\text { Portafolio } 2 \\
\text { CAN } \\
\text { dólares } \\
\text { (por ciento) }\end{array}$ & $\begin{array}{c}\text { Portafolio } 3 \\
\text { MEX pesos } \\
\text { (por } \\
\text { cieno) }\end{array}$ \\
\hline
\end{tabular}

Panel A. Mode bs univariados

\begin{tabular}{|c|c|c|c|c|}
\hline Desvest & \begin{tabular}{rr|} 
Media & $\boldsymbol{\mu}$ \\
Volatilidad & $\boldsymbol{\sigma}$ \\
& $\mathbf{R R}^{1}$
\end{tabular} & $\begin{array}{l}0.075 \\
1.467 \\
5.11\end{array}$ & $\begin{array}{c}0.040 \\
1.0301 \\
3.07\end{array}$ & $\begin{array}{l}0.068 \\
1.331 \\
5.00\end{array}$ \\
\hline \multirow{3}{*}{$E W M A$} & \multirow{3}{*}{$\begin{array}{rr}\text { Media } & \boldsymbol{\mu} \\
\text { Volatilidad } & \boldsymbol{\sigma} \\
\mathrm{RRR}\end{array}$} & $\frac{5.11}{0.060}$ & 0.080 & 0.038 \\
\hline & & 1.485 & 1.996 & 0.907 \\
\hline & & 4.04 & 4.01 & 4.22 \\
\hline \multirow{3}{*}{$\operatorname{GARCH}(1,1)^{*}$} & \multirow{3}{*}{\begin{tabular}{rr|} 
Media & $\boldsymbol{\mu}$ \\
Volatilidad & $\boldsymbol{\sigma}$ \\
RRR $^{\star}$
\end{tabular}} & 0.067 & 0.050 & 0.069 \\
\hline & & 1.180 & 1.210 & 1.011 \\
\hline & & 5.68 & 4.13 & 6.82 \\
\hline \multirow{3}{*}{ TGARCH $(1,1)$} & Media $\boldsymbol{\mu}$ & 0.067 & 0.047 & 0.068 \\
\hline & Volatilidad $\sigma$ & 1.369 & 1.230 & 1.010 \\
\hline & $\mathbf{R R R}^{*}$ & 4.47 & 3.10 & 6.65 \\
\hline
\end{tabular}

Panel B. Modelos multivariados

\begin{tabular}{|c|c|c|c|c|}
\hline \multirow{3}{*}{$D-V E C H^{*}$} & \multirow{3}{*}{\begin{tabular}{rr|} 
Media & $\boldsymbol{\mu}$ \\
Volatilidad & $\boldsymbol{\sigma}$ \\
$\mathbf{R R R}^{*}$
\end{tabular}} & 0.065 & 0.065 & 0.060 \\
\hline & & 1.451 & 1.413 & 0.936 \\
\hline & & 4.50 & 4.60 & 6.41 \\
\hline \multirow{3}{*}{$D-B E K K^{2}$} & \multirow{3}{*}{$\begin{array}{rr}\text { Media } & \boldsymbol{\mu} \\
\text { Volatilidad } & \boldsymbol{\sigma} \\
\text { RRR }^{\star}\end{array}$} & 0.040 & 0.040 & 0.040 \\
\hline & & 1.347 & 1.609 & 1.125 \\
\hline & & 297 & 2.49 & 3.56 \\
\hline \multirow{3}{*}{$C C^{*}$} & \multirow{3}{*}{$\begin{array}{|rr|}\text { Media } & \boldsymbol{\mu} \\
\text { Volatilidad } & \boldsymbol{\sigma} \\
\text { RRR }^{*}\end{array}$} & 0.070 & 0.060 & 0.064 \\
\hline & & 1.083 & 0.893 & 0.890 \\
\hline & & 6.46 & 6.72 & 7.19 \\
\hline
\end{tabular}

Nota: 1. RRR: relación riesgo-rendimiento.

*Modelos obtenidos por el método de máxima verosimilitud, así como los criterios de Akaike y Schwartz.

Lo anterior permite vislumbrar a los modelos GARCH como los mejores de acuerdo a la estimación de parámetros porque generalmente presentan resultados más consistentes en la optimación de los portafolios de inversión en las tres diferentes monedas. También es importante observar que en estos modelos los parámetros de la volatilidad (representada por $\sigma$ ) son los más bajos debido a que éstos registran los eventos raros en la distribución de sus rendimientos en comparación a los portafolios asumidos con supuestos de comportamiento normal. El modelo multivariado CCC representa en general la mejor estimación de riesgo, esto es, 1.083 para el portafolio en dólares de Estados Unidos, 0.893 para el caso del portafolio en dólares canadienses y 0.890 para 
el caso del portafolio en moneda mexicana; en este último caso, se observa una ponderación más balanceada coincidiendo con otros estudios como se demuestra en Tse y Tsui (2001), Morimoto y Kawasaki (2008), y Ortas et al. (2010).

La relación rendimiento-riesgo demuestra que México es el mercado que ofrece mayores alternativas de ganancia (7.19 por ciento) y detrás se encuentran los Estados Unidos (4.57 por ciento) y Canadá (3.83 por ciento). Esto podría explicarse por los constantes records históricos que tuvo la Bolsa Mexicana de Valores durante los años 2005, 2006 y 2007.

El cuadro 6 presenta las ponderaciones asignadas a cada portafolio de inversión una vez obtenidos los parámetros de los diferentes enfoques de media-varianza y sus correspondientes matrices de varianzas y covarianzas.

Cuadro 6. Ponderaciones de los portafolios de inversión optimizados por método de Markowitz (modelos M-VARCH). Porcentaje diario

\section{Panel A. Modelos Univariados}

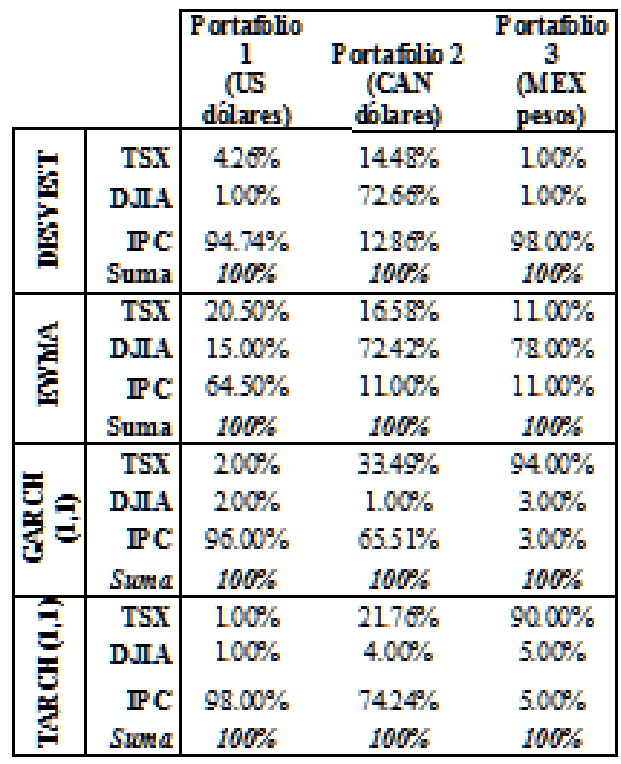

\section{Panel B. Modelos Multivariados}

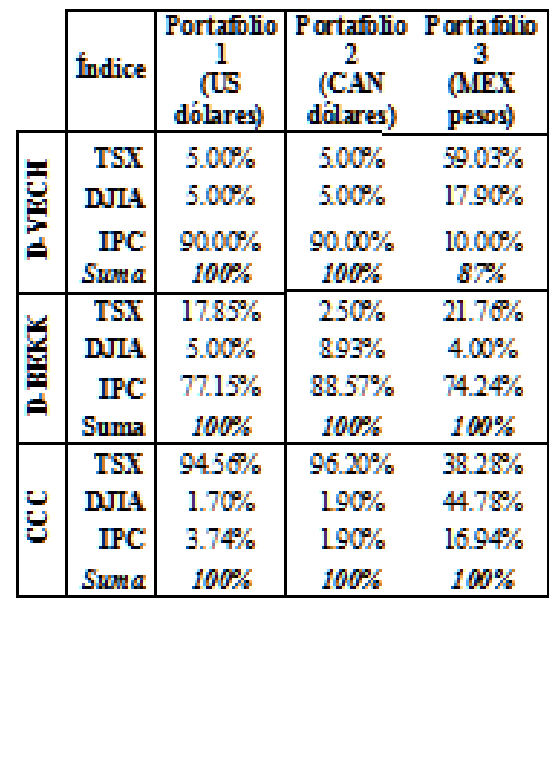

Estimación propia

El éxito de los modelos multivariados se puede explicar porque las series en conjunto capturan toda la información disponible debido a que existe una innovación $\varepsilon_{t}$ (dependiente del tiempo y vinculada a la varianza condicional) más participativa que se modifica cuando, al incorporar en la matriz de cada modelo nueva información, cambian las expectativas.

La constitución adecuada de los portafolios de inversión integrada por los diferentes métodos de volatilidad es sujeta a prueba por los modelos VaR, calculado por el método delta-normal) y posteriormente la metodología CVaR para el caso de los tres índices accionarios y tres niveles de confianza. Debido a la naturaleza del enfoque VaR condicional como medida coherente de riesgo, es posible observar que en todos los casos el método CVaR castiga un poco más el valor de la máxima pérdida esperada para cada modelo. 
El cuadro 7 permite observar los escenarios VaR y CVaR para portafolios de inversión optimizados bajo el método delta-normal para el caso de las tres economías en estudio. El panel A representa el portafolios en dólares de Estados Unidos, el panel B está representa el portafolio en dólares canadienses y el panel C representa el portafolio en pesos mexicanos. Al 99 por ciento de nivel significativo, para el caso del portafolio en dólares americanos, los modelos univariados GARCH y TARCH estiman las mayores pérdidas potenciales (3.4 por ciento y CVaR de 3.9 por ciento sobre el total de inversión, en ambos casos) y los modelos multivariados D-VECH y D-BEKK son los que reflejan mayor pérdida potencial (3.7 por ciento y CVaR de 4.2 por ciento, en ambos casos).

Cuadro 7. Estimación máxima de pérdidas bajo los enfoques VARCH univariados y multivariados en bolsas del bloque TLCAN. (Método paramétrico Delta-Normal). Portafolios optimizados.

Panel A. Portafolios en dólares (EE.UU.)

\begin{tabular}{|c|c|c|c|c|c|c|c|}
\hline \multirow{2}{*}{ Enfoque } & \multicolumn{4}{|c|}{ Unirariados } & \multicolumn{3}{|c|}{ Multivariados } \\
\hline & Desr.Est. & EWMA & $\operatorname{GARCH}(1,1)$ & $\operatorname{TARCH}(1,1)$ & D-VECH & D-BEKK & $\operatorname{ccC}$ \\
\hline VaR $99 \%$ & 0.020492 & 0.020442 & 0.034430 & 0.034849 & 0.033755 & 0.031336 & 0.025194 \\
\hline $\mathrm{CV} \mathbf{R} 9 \%$ & 0.023476 & 0.023420 & 0.039445 & 0.039925 & 0.038672 & 0.035900 & 0.028564 \\
\hline VaR $95 \%$ & 0.014459 & 0.014454 & 0.024344 & 0.024640 & 0.023867 & 0.022156 & 0.017814 \\
\hline $\mathrm{CVan} 98 \%$ & 0.018169 & 0.018126 & 0.030528 & 0.030599 & 0.029930 & 0.027785 & 0.022339 \\
\hline$v a \bar{x} 90 \%$ & 0.011288 & 0.011261 & 0.018967 & 0.019198 & 0.018595 & 0.017262 & 0.013879 \\
\hline $\mathrm{CV}$ ㄱ. $90 \%$ & 0.015459 & 0.015421 & 0.025974 & 0.026290 & 0.025465 & 0.023640 & 0.019006 \\
\hline
\end{tabular}

Panel B. Portafolios en dólares (Canadá)

\begin{tabular}{|c|c|c|c|c|c|c|c|}
\hline \multirow{2}{*}{ Enfoque } & \multicolumn{4}{|c|}{ Univariados } & \multicolumn{3}{|c|}{ Multivariados } \\
\hline & Desr. Est. & EWMA & GARCH $(1,1)$ & TARCH(1,1) & D-VECH & D-BEKK & $\mathrm{CCC}$ \\
\hline Van $99 \%$ & 0.020721 & 0.020660 & 0.028754 & 0.029987 & 0.037524 & 0.037431 & 0.020774 \\
\hline $\mathrm{CVaR} 9 \%$ & 0.023740 & 0.023669 & 0.032942 & 0.034355 & 0.042990 & 0.042883 & 0.023900 \\
\hline $\mathrm{V}=95 \%$ & 0.014651 & 0.014607 & 0.020330 & 0.021202 & 0.026531 & 0.026466 & 0.014659 \\
\hline $\mathrm{CVaR} \% \%$ & 0.018373 & 0.018318 & 0.025495 & 0.026588 & 0.033272 & 0.033189 & 0.018420 \\
\hline $\mathrm{V}=90 \%$ & 0.011415 & 0.011381 & 0.015840 & 0.016519 & 0.020671 & 0.020620 & 0.011444 \\
\hline$C V=90 \%$ & 0.015632 & 0.015586 & 0.021692 & 0.022622 & 0.028308 & 0.028238 & 0.015672 \\
\hline
\end{tabular}

Panel C. Portafolios en pesos (México)

\begin{tabular}{|c|c|c|c|c|c|c|c|}
\hline \multirow{2}{*}{ Enfoque } & \multicolumn{4}{|c|}{ Unirariados } & \multicolumn{3}{|c|}{ Multirariados } \\
\hline & Desr.Est. & EWMA & GARCH $(1,1)$ & $\operatorname{TARCH}(1,1)$ & D-VECH & D-BEKK & $\mathrm{CCC}$ \\
\hline $\operatorname{Var} 99 \%$ & 0.020721 & 0.020659 & 0.024217 & 0.023799 & 0.021775 & 0.026171 & 0.020635 \\
\hline $\mathrm{CVaR} 99 \%$ & 0.023740 & 0.023669 & 0.027745 & 0.027265 & 0.024946 & 0.029984 & 0.023640 \\
\hline $\mathrm{VaR} 95 \%$ & 0.014651 & 0.014607 & 0.017123 & 0.016527 & 0.015396 & 0.018505 & 0.014590 \\
\hline CVan $95 \%$ & 0.018373 & 0.018318 & 0.021473 & 0.021102 & 0.019307 & 0.023206 & 0.01829 \\
\hline $\operatorname{Var} 90 \%$ & 0.011415 & 0.011381 & 0.013341 & 0.013110 & 0.011995 & 0.014417 & 0.01136 \\
\hline $\mathrm{CVaR} 90 \%$ & 0.015632 & 0.015585 & 0.018269 & 0.017953 & 0.016427 & 0.019744 & 0.01536 \\
\hline
\end{tabular}

Estimación propia 
150 Nueva Época REMEF (The Mexican Journal of Economics and Finance)

En México el modelo D-BEKK es el modelo que mayores pérdidas potenciales diarias presenta (2.6 por ciento y CVaR de 2.9 por ciento). Similares casos se presentan a los niveles significativos de confianza del 95 y 90 por ciento. También es de llamar la atención cómo los modelos multivariados presentan los resultados más conservadores al registrar las mayores pérdidas potenciales del total de medidas de riesgo paramétricas estudiadas en los diferentes niveles de significancia. Finalmente, se aprecia también que los modelos normales desvest (desviación estándar) y EWMA representan el grado más bajo de pérdidas resultando en estimaciones menos conservadoras para el análisis del riesgo financiero.

Los paneles A, B y C del cuadro 8 presentan la estimación del Valor en Riesgo mediante tres métodos no paramétricos (conocidos como simulación histórica). El panel A muestra el método de simulación histórica en dólares americanos; el panel $\mathrm{B}$ muestra los resultados del portafolio en dólares canadienses; por último, el panel $\mathrm{C}$ muestra el portafolio en pesos mexicanos. En los tres paneles es posible apreciar que la máxima pérdida potencial estimada se presenta bajo el método de crecimientos absolutos, sobretodo en el caso de los modelos GARCH; incluso los resultados son mayores que el cálculo representado por la metodología paramétrica del cuadro 7; esto podría representar un error debido a que los métodos no paramétricos pueden mostrar resultados fuera del rango en cada uno de los modelos con respecto a la proporción presentada por las estimaciones cuantificadas de forma paramétrica. Esto es, los métodos no paramétricos subestiman o sobreestiman el nivel de máxima pérdida esperada para el periodo de tiempo establecido. Por ejemplo, en el caso del método de simulación histórica por crecimientos absolutos (CA), en su valuación GARCH y TARCH, cuyos resultado son de 5.2 por ciento para ambos casos al nivel del 99 por ciento significativo, contra el 2.8 por ciento de las técnicas (CL) y (CR) CCC multivariado, es decir, casi doblemente mayor a los resultados mostrados en el cuadro 5 . 
Cuadro 8. Pérdidas máximas, enfoques VARCH Univariados y multivariados paramétricos de simulación histórica. Ajuste por la metodología GARCH.

Modelos Unirariados

Panel A. Portafolios en dólares (EE.UU.)

\begin{tabular}{|r|ccc|ccc|ccc|ccc|}
\hline Métcolo" & \multicolumn{3}{|c|}{ Desr.Est } & \multicolumn{3}{c|}{ ETMA } & \multicolumn{3}{c|}{ GARCH } & \multicolumn{3}{c|}{ TARCH } \\
Percentil & CA & CL & CR & CA & CL & CR & CA & CL & CR & CA & CL & CR \\
\hline $99 \%$ & 0.019 & 0.026 & 0.027 & 0.019 & 0.026 & 0.027 & 0.053 & 0.029 & 0.029 & 0.053 & 0.029 & 0.029 \\
$95 \% / 20.007$ & 0.014 & 0.014 & 0.007 & 0.014 & 0.014 & 0.040 & 0.017 & 0.017 & 0.040 & 0.017 & 0.017 \\
$90 \% / 0.003$ & 0.010 & 0.010 & 0.003 & 0.010 & 0.010 & 0.035 & 0.012 & 0.012 & 0.035 & 0.012 & 0.012 \\
\hline
\end{tabular}

Modelos Multir ariados

\begin{tabular}{|r|ccccccccc|}
\hline Métodlo & \multicolumn{3}{|c}{ D-VECH } & \multicolumn{3}{c|}{ D-BEKK } & & CCC \\
Percenti & CA & CL & CR & CA & CL & CR & CA & CL & CR \\
\hline $99 \% / 20.017$ & 0.024 & 0.024 & 0.017 & 0.024 & 0.024 & 0.017 & 0.024 & 0.024 \\
$95 \% / 2$ & 0.007 & 0.013 & 0.013 & 0.007 & 0.013 & 0.013 & 0.007 & 0.013 & 0.013 \\
$90 \% / 8$ & 0.003 & 0.010 & 0.010 & 0.003 & 0.010 & 0.010 & 0.003 & 0.010 & 0.010 \\
\hline
\end{tabular}

Modelos Unirariados

Panel B. Portafolios en dólares (Canadá)

\begin{tabular}{|r|ccc|ccc|ccc|ccc|}
\hline Mémod & \multicolumn{3}{|c|}{ Desr.Est. } & \multicolumn{3}{|c|}{ EWMA } & \multicolumn{3}{|c|}{ GARCH } & \multicolumn{3}{|c|}{ TGARCH } \\
Percentil & CA & CL & CR & CA & CL & CR & CA & CI & CR & CA & CL & CR \\
\hline $99 \% / 8$ & 0.019 & 0.026 & 0.027 & 0.019 & 0.026 & 0.027 & 0.053 & 0.030 & 0.03 & 0.053 & 0.030 & 0.030 \\
$95 \% / 8$ & 0.007 & 0.014 & 0.014 & 0.007 & 0.014 & 0.014 & 0.039 & 0.016 & 0.016 & 0.039 & 0.016 & 0.016 \\
$90 \% / 2$ & 0.003 & 0.010 & 0.010 & 0.003 & 0.010 & 0.010 & 0.034 & 0.011 & 0.011 & 0.034 & 0.011 & 0.011 \\
\hline
\end{tabular}

\begin{tabular}{|c|c|c|c|c|c|c|c|c|c|}
\hline \multicolumn{10}{|c|}{ Modelos Multir ariados } \\
\hline \multirow{2}{*}{$\begin{array}{l}\text { Métob } \\
\text { Percentil }\end{array}$} & \multicolumn{3}{|c|}{ D-VECH } & \multicolumn{3}{|c|}{ BEKK } & \multicolumn{3}{|c|}{$\mathrm{CCC}$} \\
\hline & CA & CL & CR & CA & CL & CR & CA & CL & CR \\
\hline $99 \%$ & 0.018 & 0.023 & 0.024 & 0.018 & 0.023 & 0.024 & 0.018 & 0.023 & 0.024 \\
\hline $95 \%$ & 0.008 & 0.013 & 0.013 & 0.008 & 0.013 & 0.013 & 0.008 & 0.013 & 0.013 \\
\hline $90 \%$ & 0.004 & 0.010 & 0.010 & 0.004 & 0.010 & 0.010 & 0.004 & 0.010 & 0.010 \\
\hline
\end{tabular}

Panel C. Portafolios en pesos (México)

\begin{tabular}{|c|c|c|c|c|c|c|c|c|c|}
\hline \multirow{2}{*}{$\begin{array}{c}\text { Métodb } \\
\text { Percentil }\end{array}$} & \multicolumn{3}{|c|}{ Desr. Est. } & \multicolumn{3}{|c|}{ EWMA } & \multicolumn{3}{|c|}{ GARCH } \\
\hline & CA & CL & CR & CA & $C L$ & CR & CA & $\mathrm{CI}$ & CR \\
\hline $99 \%$ & 0.019 & 0.026 & 0.027 & 0.019 & 0.026 & 0.027 & 0.051 & 0.028 & 0.028 \\
\hline $95 \%$ & 0.007 & 0.014 & 0.014 & 0.007 & 0.014 & 0.014 & 0.039 & 0.016 & 0.016 \\
\hline $90 \%$ & 0.003 & 0.010 & 0.010 & 0.003 & 0.010 & 0.010 & 0.034 & 0.011 & 0.011 \\
\hline \multicolumn{10}{|c|}{ Modelos Multir ariados } \\
\hline Mébab* & \multicolumn{3}{|c|}{ D-VECH } & \multicolumn{3}{|c|}{ BEKK } & \multicolumn{3}{|c|}{$\mathrm{CCC}$} \\
\hline Percentil & CA & $\mathrm{CL}$ & CR & CA & CL & CR & CA & CL & CR \\
\hline $99 \%$ & 0.018 & 0.023 & 0.024 & 0.018 & 0.023 & 0.024 & 0.018 & 0.026 & 0.027 \\
\hline $95 \%$ & 0.008 & 0.013 & 0.013 & 0.008 & 0.013 & 0.013 & 0.008 & 0.014 & 0.014 \\
\hline $90 \%$ & 0.004 & 0.010 & 0.010 & 0.004 & 0.010 & 0.010 & 0.004 & 0.010 & 0.010 \\
\hline
\end{tabular}

Estimación propia 


\subsection{Análisis retrospectivo (Backtesting)}

Una vez evaluadas las pérdidas potenciales es imperativo evaluar la precisión de las alternativas de medición del riesgo VaR y CVaR. Para tal efecto se desarrolla el análisis retrospectivo empleando una muestra de 250 datos (recomendado por el Comité de Basilea). La muestra comprende el periodo 2007-01-16 a 2007-12-31. En primer lugar, se debe demostrar que la metodología planteada es válida, o bien, que no puede ser sujeta de rechazo dado su implementación, i.e., los valores resultantes de esta prueba son coherentes. En segundo lugar, suponiendo verdadero el punto anterior para el caso de series de índices bursátiles de los países que integran el TLCAN, se debe encontrar que entre los enfoques VaR utilizados, existe aquél que mejor refleje el riesgo de mercado en portafolios de inversión.

El cuadro 7 muestra, en las tres divisas del bloque del TLCAN, la estimación de máximas pérdidas bajo los enfoques VARCH univariados y multivariado aplicando el método paramétrico delta-normal. Al nivel de significancia del 99, 95 y 90 por ciento, los modelos multivariados pasan la prueba. Los modelos normalizados desv.est., EWMA, además del modelo GARCH $(1,1)$ presentan problemas en su desempeño, lo cual sugiere un ajuste a un nivel significativo menor para la re-calibración de los modelos. En general, el desempeño mostrado por los modelos multivariados permite realizar una mejor estimación en los modelos VaR y obtener portafolios de inversión confiables en cualquiera de los tres países en donde se desee invertir en moneda local. En todos los casos se observa la manera en la cual, a medida que el nivel de significancia se aproxima al cien por ciento, existe mayor posibilidad de que los excesos sean menores al conservarse en la banda de confianza. Por ejemplo, en los modelos multivariados presentan la menor probabilidad de fracasos al 95 por ciento (siete en cada caso). Evidentemente los portafolios deben ser reevaluados cotidianamente para mantener las expectativas de pérdidas y ganancias con baja probabilidad de errores.

Los paneles A, B y C del cuadro 8 presentan la estimación del Valor en Riesgo mediante tres métodos no paramétricos (conocidos como simulación histórica). El panel A muestra el método de simulación histórica en dólares americanos; el panel $\mathrm{B}$ muestra los resultados del portafolio en dólares canadienses; por último, el panel $\mathrm{C}$ muestra el portafolio en pesos mexicanos. En los tres paneles es posible apreciar que la máxima pérdida potencial estimada se presenta bajo el método de crecimientos absolutos, sobretodo en el caso de los modelos GARCH; incluso los resultados son mayores que el cálculo representado por la metodología paramétrica del cuadro 7; esto podría representar un error debido a que los métodos no paramétricos pueden mostrar resultados fuera del rango en cada uno de los modelos con respecto a la proporción presentada por las estimaciones cuantificadas de forma paramétrica. Esto es, los métodos no paramétricos subestiman o sobreestiman el nivel de máxima pérdida esperada para el periodo de tiempo establecido. Por ejemplo, en el caso del método de simulación histórica por crecimientos absolutos (CA), en su valuación GARCH y TARCH, cuyos resultado son de 5.2 por ciento para ambos casos al nivel del 99 por ciento significativo, contra el 2.8 por ciento de las técnicas (CL) y (CR) CCC multivariado, es decir, casi doblemente mayor a los resultados mostrados en el cuadro 5 . 
Finalmente, el cuadro 9 muestra la prueba de proporción de fallas de Kupiec. El panel A se presentan los modelos univariados y en el panel B los modelos multivariados. El apéndice A muestra diversas regiones de no rechazo para un número determinado de observaciones fuera del VaR propuesto por el Comité de Basilea para el análisis retrospectivo. Dos conclusiones deben extraerse al llevar a cabo del backtesting. Al nivel significativo del 99, 95 y 90 por ciento, los modelos multivariados pasan la prueba. Los modelos normalizados desv.est., EWMA, además del modelo GARCH $(1,1)$ presentan problemas en su desempeño, lo cual sugiere un ajuste a un nivel significativo menor para la re-calibración de los modelos. En general, el desempeño mostrado por los modelos multivariados permite realizar una mejor estimación en los modelos VaR y obtener portafolios de inversión confiables en cualquiera de los tres países en donde se desee invertir en moneda local. En todos los casos se observa la manera en la cual, a medida que el nivel de significancia se aproxima al cien por ciento, existe mayor posibilidad de que los excesos sean menores al conservarse en la banda de confianza. Por ejemplo, en los modelos multivariados presentan la menor probabilidad de fracasos al 95 por ciento (siete en cada caso). Evidentemente los portafolios deben ser reevaluados cotidianamente para mantener las expectativas de pérdidas y ganancias con baja probabilidad de errores.

Cuadro 9. Prueba de proporción de fallas (Backtesting). Método de Kupiec ${ }^{1}$ Aplicación a portafolios VaR (delta-garch) del TLCAN ${ }^{2}$.

Panel A. Mode los univariados

\begin{tabular}{|r|c|cccc|}
\hline $\begin{array}{r}\text { Modelo de } \\
\text { rolatilidad }\end{array}$ & $\begin{array}{c}\text { VaR } \\
(0 \%)\end{array}$ & $\mathbf{X}^{(b)}$ & (a) & (b) & (c) \\
Desw. Est. & 99 & 12 & .0480 & 19.020 & 0.0006 \\
EWMLA & 99 & 12 & .0560 & 15.368 & 0.0005 \\
GARCH(1,1) & 99 & 10 & 4036 & 12955 & 0.0003 \\
T.ARCH(1,1) & 99 & 2 & 0.402 & 0.1084 & 0.7419 \\
\hline Desw. Est. & 95 & 29 & 11.6 & 19.02 & 0.0059 \\
EWMLA & 95 & 27 & 10.3 & 13.69 & 0.0063 \\
GARCH(1,1) & 95 & 24 & 9.6 & 8.8776 & 0.0028 \\
T.ARCH(1,1) & 95 & 11 & 0.38 & 0.1971 & 0.6571 \\
\hline Desv. Est. & 90 & 41 & 16.34 & 9.7300 & 0.1800 \\
EWMLA & 90 & 41 & 16.00 & 8.9700 & 0.0486 \\
GARCH(1,1) & 90 & 40 & 16.00 & 8.0232 & 0.0033 \\
T.ARCH(1,1) & 90 & 17 & 0.36 & 3.1686 & 0.0751 \\
\hline
\end{tabular}

Panel B. Modelos multivariados

\begin{tabular}{|rc|cccc|}
\hline $\begin{array}{r}\text { Modelode } \\
\text { rolatilidad }\end{array}$ & $\begin{array}{c}\text { VaR } \\
(9 \%)\end{array}$ & $\mathbf{x}^{(\text {t) }}$ & (a) & (b) & (c) \\
D.VECH & 99 & 0 & N.A. & N.A. & N.A. \\
D-BEKK & 99 & 0 & N.A. & N.A. & N.A \\
CCC & 99 & 1 & 0.4000 & 1.1765 & 0.2781 \\
D.VECH & 95 & 7 & 0.3809 & 3.0456 & 0.0828 \\
D-BEKK & 95 & 7 & 0.3801 & 3.0323 & 0.0828 \\
CCC & 95 & 7 & 0.3803 & 3.0080 & 0.0828 \\
D.VECH & 90 & 21 & 0.3636 & 0.7479 & 0.3872 \\
D-BEKK & 90 & 20 & 0.3687 & 1.1846 & 0.2764 \\
CCC & 90 & 21 & 0.3655 & 0.7479 & 0.3872 \\
\hline
\end{tabular}

Notas: 1) Hipótesis nula (Ho): La proporción de fallas es similar a la significancia estadística propuesta en el modelo (¡0.05). N.A.: No aplicable.

\section{Conclusiones}

Este documento propone una metodología aplicada a portafolios internacionales de inversión bajo el enfoque M-VARCH. Con el fin de evaluar la capacidad de esta metodología, se compara con otros enfoques paramétricos habituales para el cálculo de la volatilidad condicional con aplicación a tres portafolios conformados por tres índices accionarios representativos de los países que conforman el bloque regional del TLCAN y denominados tanto en dólares como en la moneda local. La potencia de 
los modelos multivariados es plenamente confirmada. Es posible concluir que mediante el uso de la metodología VARCH (aplicación de los métodos GARCH a los modelos de Valor en Riesgo, VaR) en estos mercados producen mejores estimados sobre pérdidas potenciales para los inversionistas locales y extranjeros que con la cuantificación de riesgos mediante la metodología delta-normal tradicional. En el caso particular de México, la evidencia sugiere que la diversificación hacia este mercado es una opción atractiva debido a que con sus altos rendimientos es posible conformar portafolios trinacionales que minimicen el riesgo. No obstante, a mayor integración de estos mercados los beneficios de la diversificación tenderían a disminuir en este bloque.

\section{Apéndice A.}

Método de Kupiec. Región de no rechazo para el número de observaciones $(\mathrm{N})$ fuera del VaR

\begin{tabular}{|c|c|c|c|c|}
\hline $\begin{array}{r}\text { Niv } \\
\text { probat }\end{array}$ & $\begin{array}{l}\text { I de } \\
\text { lidad, }\end{array}$ & $\begin{array}{c}\mathrm{T}=25 \mathrm{~s} \\
\text { dias }\end{array}$ & $\begin{array}{c}T=510 \\
\text { dís }\end{array}$ & $\begin{array}{c}\mathrm{T}=1,000 \\
\text { dias }\end{array}$ \\
\hline 0.001 & 0.196 & $\mathrm{~N}<7$ & $10 \mathrm{~N}<1$ & $40 \mathrm{~N}<17$ \\
\hline 0.025 & 2.596 & $29 \mathrm{~N}<12$ & $60 \mathrm{Nol}$ & $150 \mathrm{~N} 36$ \\
\hline 0.05 & 546 & $60 \mathrm{~N} 21$ & $16 \bigcirc \bigcirc 6$ & $37 \bigcirc \times 65$ \\
\hline 0.075 & $7.5 \%$ & $11 \mathrm{~N} 88$ & $827 @<51$ & $590 \times<2$ \\
\hline 0.10 & $10 \%$ & $169 \mathrm{QPB}$ & & \\
\hline
\end{tabular}

Fuente: De Lara (2001).

\section{Bibliografía}

Alonso, J. y M. Arcos (2006). Valor en Riesgo: Evaluación del Desempeño de Diferentes Metodologías para 7 Países Latinoamericanos. Borrador de Economía, Universidad ICESI, Colombia.

Angelidis, T. and Benos, A. (2003). The Use of GARCH Models in VaR Estimation. Working Paper, Dept. of Banking and Financial Management, Universitty of Piraeus, Grecia.

Bauwens, L., S. Laurent, and J. Rombouts (2006). Multivariate GARCH Models: A Survey. Journal of Applied Econometrics, 21, pp. 79-109.

Bollerslev, T. (1986), Generalized Autoregressive Conditional Heteroskedasticity. Journal of Econometrics, 31, pp. 307, 327.

Bollerslev, T., R. F. Engle and J. Wooldridge (1988). A Capital Asset Pricing Model with Time-Varying Covariances. Journal of Political Economy, 96(1), pp. 116-131.

Bollerslev, T. (1990). Modelling the Coherence in Short-Run Nominal Exchange Rates: A Multivariate Generalized ARCH Model. Review of Economics and Statistics, 72, pp.498-505. 
Burns, P. (2002). The Quality of Value at Risk via Univariate GARCH. Working Paper. Burns Statistics, Londres.

De Jesús, R. and E. Ortiz (2012). Risk in Emerging Stock Markets from Brazil and Mexico: Extreme Value Theory and Alternative Value at Risk Models. Frontiers in Finance and Economics, 8(2), pp. 49-88.

De Lara, A. (1995). Control y Medición de Riesgos Financieros. Limusa, México, 2002.

Engle, R.F. and K. Kroner (1995). Multivariate Simultaneous GARCH. Econometric Theory 11, pp. 122-150.

Fernández, Á., V. Aragó (2002). Transmisión de la Volatilidad entre los Mercados Bursátiles Desde la Perspectiva Española. Working Paper. Universidad Jaume I, España.

Gallón, S. y K. Gómez (2007). Distribución Condicional de los Retornos de la Tasa de Cambio Colombiana: Un Ejercicio Empírico a Partir de Modelos GARCH Multivariados. Revista de Economía del Rosario, 10(2), Bogotá, pp.127-152.

González, S. y J. Mascareñas (1999). La globalización de los mercados financieros, Noticias de la Unión Europea, 172, Universidad Complutense de Madrid, pp. 15-35.

Korkmaz, T y Aidin, K. (2002). Using EWMA and GARCH Methods in VaR calculations: Application to ISE-30 Index. Working Paper, Zonguldak Karaelmas University (Turquía).

Kupiec, P., (1995). Techniques for Verifying the Accuracy of Risk Measurement Models. Finance and Economics Discussion Series, Federal Reserve Board, EE.UU., pp. 95124.

López Herrera, F., E. Ortiz y A. Cabello (2009). Las Interrelaciones de Volatilidad y Rendimientos entre los Mercados de Valores del TLCAN. Investigación Económica, vol. LXVIII, enero-marzo, pp. 83-114.

López Herrera, F. (2006). Riesgo Sistemático en el Mercado Mexicano de Capitales: Un Caso de Segmentación Parcial. Revista Contaduría y Administración, UNAM, México, 269, pp. 85-113.

Ludlow, J. y B. Mota (2006).Volatilidad del IPC, Nasdaq y S\&P 500: Un Modelo GARCH Multivariado. Revista Análisis Económico, 21, pp. 217-227.

Morimoto, T. and Y. Kawasaki (2008). Empirical Comparison of Multivariate GARCH Models for Estimation of Intraday Value at Risk. Working Paper. Hitotsubashi University.

Markowitz, H.W. (1952), Portfolio Selection. The Journal of Finance, 7, pp. 77-91.

Markowitz, H.W. (1959). Portfolio Selection: Efficient Diversification of Investments. New York, John Wiley \& Sons.

Ortas E., J. Moneva, y M. Salvador (2010). Conditional Volatility in Sustainable and Traditional Stock Exchange Indexes: Analysis of the Spanish Market. Globalization, Competitiveness \& Governability, Georgetown University, US, pp. 104-129.

Ortiz, E., A. Cabello y F. López Herrera(2007a). Mercados de capital emergentes y desarrollo e integración. En Girón, Alicia; Correa, Eugenia, Del Sur hacia el Norte: Economía Política del Orden Económico Internacional Emergente, CLACSO, Argentina.

Ortiz, E., A. Cabello y F. López Herrera (2007b). Las Bolsas de Valores en el TLCAN: Un Análisis a Largo Plazo. Revista Problemas del Desarrollo, 38(151), pp. 37-61.

Ramírez, E., y P. Ramírez (2007). Valor en Riesgo: Modelos Econométricos contra Metodologías Tradicionales. Revista Análisis Económico, 22, pp. 179-198.

Tagliafichi, R. (2003), The Estimation of Market VaR using GARCH Models and Heavy Tail Distributions. AFIR colloquium, Holanda.

Tse, Y.K.y A. Tsui (2001). A Multivariate GARCH Model with Time-Varying Correlations. Econometric Society World Congress 2000.

Uryasev S. y R. Rockafellar (2002). Conditional Value-at-Risk for General Loss Distributions. Journal of Banking and Finance, 26(7), pp. 1443-1471.

Vergara, M. y C. Maya (2009). Monte Carlo Estructurado. Estimación del Valor en Riesgo en un Portafolio Accionario en Colombia. Administer, Universidad EAFIT, Colombia, 15, pp. $68-88$. 